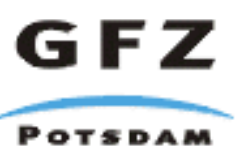

Originally published as:

Ring, U., Johnson, C., Hetzel, R., Gessner, K. (2003): Tectonic denudation of a Late CretaceousTertiary collisional belt: Regionally symmetric cooling patterns and their relation to extensional faults in the Anatolide belt of western Turkey. - Geological Magazine, 140, 4, 421-441

DOI: $10.1017 / \mathrm{S} 0016756803007878$ 


\title{
Tectonic denudation of a Late Cretaceous-Tertiary collisional belt: regionally symmetric cooling patterns and their relation to extensional faults in the Anatolide belt of western Turkey
}

\author{
UWE RING*, CHRISTOPHER JOHNSON $\dagger$, RALF HETZEL $\ddagger \&$ KLAUS GESSNER*§ \\ *Institut für Geowissenschaften, Johannes Gutenberg-Universität, 55099 Mainz, Germany \\ $\dagger$ †epartment of Earth Science and Engineering, Imperial College, London SW7 2BP, UK \\ $\ddagger$ GeoForschungsZentrum, 14473 Potsdam, Germany
}

(Received 3 October 2002; accepted 24 March 2003)

\begin{abstract}
Thermochronological data reveal that the Late Cretaceous-Tertiary nappe pile of the Anatolide belt of western Turkey displays a two-stage cooling history. Three crustal segments differing in structure and cooling history have been identified. The Central Menderes metamorphic core complex represents an 'inner' axial segment of the Anatolide belt and exposes the lowest structural levels of the nappe pile, whereas the two 'outer' submassifs, the Gördes submassif to the north and the Çine submassif to the south, represent higher levels of the nappe pile. A regionally significant phase of cooling in the Late Oligocene and Early Miocene affected the outer two submassifs and the upper structural levels of the Central Menderes metamorphic core complex. In the northern part of the Gördes submassif, cooling was related to top-to-the-NNE movement on the Simav detachment, as the apatite fission-track ages show a northward-younging trend in the direction of movement on this detachment. In the Çine submassif, relatively rapid cooling in Late Oligocene and Early Miocene times may have been related to top-to-the-S extensional reactivation of the basal thrust of the overlying Lycian nappes. The second phase of cooling in the Anatolide belt is related to Pliocene to Recent extension resulting in the formation of the Central Menderes metamorphic core complex in the inner part of the Anatolide belt. Core-complex development caused the formation of supra-detachment graben, which document the ongoing separation of the Central Menderes metamorphic core complex from the outer submassifs.
\end{abstract}

Keywords: fission-track dating, exhumation, extension, Anatolides, Turkey.

\section{Introduction}

The Anatolide belt of western Turkey (also referred to as the Menderes Massif) and the adjacent Aegean Sea underwent regional extensional deformation from at least Oligocene times to the present (Lister; Banga \& Feenstra, 1984; Buick, 1991; Seyitoğlu, Scott \& Rundle, 1992; Hetzel et al. 1995a; Gessner et al. 2001a). In the Aegean, extension is controlled by southward retreat of the subducting Hellenic slab, and displacement on major extensional detachments is of the order of 50 to more than $100 \mathrm{~km}$ (Lister \& Forster, 1996; Ring, Layer \& Reischmann, 2001; Ring, Brachert \& Fassoulas, 2001). In contrast, no largemagnitude extensional detachments have been mapped so far in the Anatolide belt of western Turkey and extension is apparently not controlled by slab retreat (Hetzel et al. 1995b; Ring et al. 1999a). While both the Anatolide belt and the Aegean are characterized by southward propagation of crustal shortening, the

\footnotetext{
* Author for correspondence: ring@uni-mainz.de

$\S$ Present address: CSIRO Exploration and Mining, Bentley, WA 6102, Australia
}

geometric pattern of extensional faults and the overall magnitude of extension differ fundamentally in both areas.

In this article we report on the results of a reconnaissance fission-track study across the Anatolide belt of western Turkey. Fission-track thermochronology is a powerful tool for establishing regional variations in the cooling history of an orogen (Wagner \& Reimer, 1972; Johnson, Harbury \& Hurford, 1997; Foster \& John, 1999). Combining regional cooling patterns with structural analysis can deliver important constraints to the exhumation history of an orogen (Hurford, Flisch \& Jäger, 1989; Wheeler \& Butler, 1994; Brandon, Roden-Tice \& Garver, 1998). We will show that the regional pattern of apatite fission-track ages is remarkably symmetric at the orogen scale, with the oldest (Late Oligocene to Early Miocene) cooling ages occurring in the southern and northern margins and younger (Late Miocene to Quaternary) cooling ages in the centre of the belt. When fission-track data are combined with ${ }^{40} \mathrm{Ar} /{ }^{39} \mathrm{Ar}$-mica ages, two distinct periods of normal faulting are resolved, which have exhumed the Anatolide belt from mid-crustal depths since Late Oligocene times. 


\section{Tectonic setting}

\section{2.a. Hellenide-Anatolide orogen}

The Late Cretaceous-Tertiary Hellenide-Anatolide orogen in the eastern Mediterranean shows significant along-strike variations in basement and structure (Fig. 1). Single-zircon ${ }^{207} \mathrm{~Pb} /{ }^{206} \mathrm{~Pb}$ evaporation, conventional $\mathrm{U} / \mathrm{Pb}$-multi-grain and $\mathrm{U} / \mathrm{Pb}$-SHRIMP dating of magmatic zircons yielded Carboniferous protolith ages of $\sim 300-320 \mathrm{Ma}$ for large parts of the preAlpine basement of the Hellenide belt (S. Keay, unpub. Ph.D. thesis, Australian Nat. Univ., 1998; Reischmann, 1998; Engel \& Reischmann, 1998; Ring, Laws \& Bernet, 1999; Reischmann et al. 2001). In contrast, the pre-Alpine basement in the structurally deepest tectonometamorphic unit of the Anatolide belt in western Turkey, the Menderes nappes, yielded ages of $\sim 530-560 \mathrm{Ma}$, and Carboniferous ages are conspicuously absent (Hetzel \& Reischmann, 1996; C. Dannat, unpub. Ph.D. thesis, Univ. Mainz, 1997; Hetzel et al. 1998; Loos \& Reischmann, 1999; Gessner et al. 2001b, 2003). Therefore, Ring et al. (1999a) and Gessner et al. (2001c) regarded the Menderes nappes as part of the exotic Anatolide continent, or MenderesTauride block of Robertson \& Dixon (1984), in the Hellenide-Anatolide orogen.

Late Cretaceous to Tertiary subduction and accretion processes in both regions were also different. In the Aegean, subduction, associated high-pressure metamorphism and the formation of southward-propagating magmatic arcs and back-arc basins took place from Late Cretaceous times until the present (Fytikas et al. 1984; Ring \& Layer, 2003). In western Turkey, subduction and associated high-pressure metamorphism only occurred from Late Cretaceous to Eocene times (Gessner et al. 2001c). It has been proposed that the collision of the Anatolian continent in the Eocene halted subduction and the development of magmatic arcs and back-arc basins in western Turkey (Hetzel et al. 1995b; Collins \& Robertson, 1998; Ring et al. 1999a).

The Anatolide belt in western Turkey comprises three major tectonometamorphic units (Figs 1,2) which are characterized by different lithologies, protolith ages, pre-collisional palaeogeography and orogenic history (Ring et al. 1999a). This heterogeneous tectonic pile was assembled during Eocene collisionrelated crustal shortening. The Lycian nappes and the Izmir-Ankara suture zone represent the uppermost tectonometamorphic unit, which was deformed and, at least in part, metamorphosed under high-pressure conditions during Late Cretaceous times (before $\sim 75 \mathrm{Ma}$ ) (Collins \& Robertson, 1997; Sherlock et al. 1999; Oberhänsli et al. 2001; Ring \& Layer, 2003). The middle tectonometamorphic unit is correlative with the Cycladic blueschist unit in the Aegean (Candan et al. 1997; Ring et al. 1999a). In the Eocene, the Cycladic blueschist unit was emplaced onto the Menderes nappes along the Cyclades-Menderes thrust (Fig. 1) during the greenschist-facies $\mathrm{D}_{\mathrm{A} 3}$ event. Because the $\mathrm{D}_{\mathrm{A} 3}$ emplacement of Late Cretaceous-Tertiary highpressure units onto the Menderes nappes did not cause a high-pressure overprint in the latter, the upper and middle units must have been already exhumed to midcrustal levels by Eocene times (Ring et al. 1999a; Gessner et al. 2001c).

\section{2.b. The Menderes nappes}

Samples for this study were collected from the Menderes nappes, which form the structurally lowest tectonometamorphic unit of the Anatolide belt. The Menderes nappes comprise from top to bottom: (1) the Selimiye nappe, (2) the Çine nappe, (3) the Bozdağ nappe, and (4) the Bayındır nappe. The Çine and Bozdağ nappes have a polyorogenic history, which extends back into the Neoproterozoic/Cambrian (Gessner et al. 2001b,c; Candan et al. 2001; Ring, Willner \& Lackmann, 2001).

The Selimiye nappe contains Palaeozoic metapelite, metabasite and marble (Schuiling, 1962; Çağlayan et al. 1980; Loos \& Reischmann, 1999; Régnier et al. 2003). Most of the Çine nappe consists of deformed orthogneiss, largely undeformed metagranite and minor pelitic gneiss, eclogite and amphibolite. Protoliths of much of the orthogneiss/metagranite intruded at $\sim 530-560 \mathrm{Ma}$ (see previous Section). The underlying Bozdağ nappe is made up of metapelite with intercalated amphibolite, eclogite and marble lenses. Protolith ages of all rock types of the Bozdağ nappe are unknown, but geological constraints (Candan et al. 2001; Gessner et al. 2001b) suggest a Precambrian age for at least parts of these rocks. The Bozdağ nappe was intruded by granitoids at $230-240 \mathrm{Ma}$ (C. Dannat, unpub. Ph.D. thesis, Univ. Mainz, 1997). The Bayındır nappe contains phyllite, quartzite, marble and greenschist of inferred Permo-Carboniferous and possibly Mesozoic age (O. Candan, pers. comm. 1999). The rocks were affected by a single Tertiary greenschist-facies metamorphism during the $\mathrm{D}_{\mathrm{A} 3}$ event. ${ }^{40} \mathrm{Ar} /{ }^{39} \mathrm{Ar}$ ages of $~ 38-36 \mathrm{Ma}$ (Lips et al. 2001) suggest an Eocene age for this event.

$\mathrm{D}_{\mathrm{A} 3}$ produced a variably spaced mylonitic shearband foliation and a stretching lineation associated with top-to-the-S kinematic indicators. $\mathrm{D}_{\mathrm{A} 3}$ shear zones define the boundaries between the Selimiye, Çine, Bozdağ and Bayındır nappes, including the top-to-theS Selimiye shear zone, which separates the Selimiye nappe from the underlying Çine nappe. Bozkurt \& Park (1994) interpreted the Selimiye shear zone as a Late Oligocene top-to-the-S extensional detachment. Hetzel \& Reischmann (1996) showed that ${ }^{40} \mathrm{Ar} /{ }^{39} \mathrm{Ar}$ muscovite ages of 35-43 Ma constrain slow Eocene cooling during and after movement at the Selimiye shear zone. Subsequently, Ring et al. (1999a), Gessner et al. (2001c) and Régnier et al. (2003) supplied 


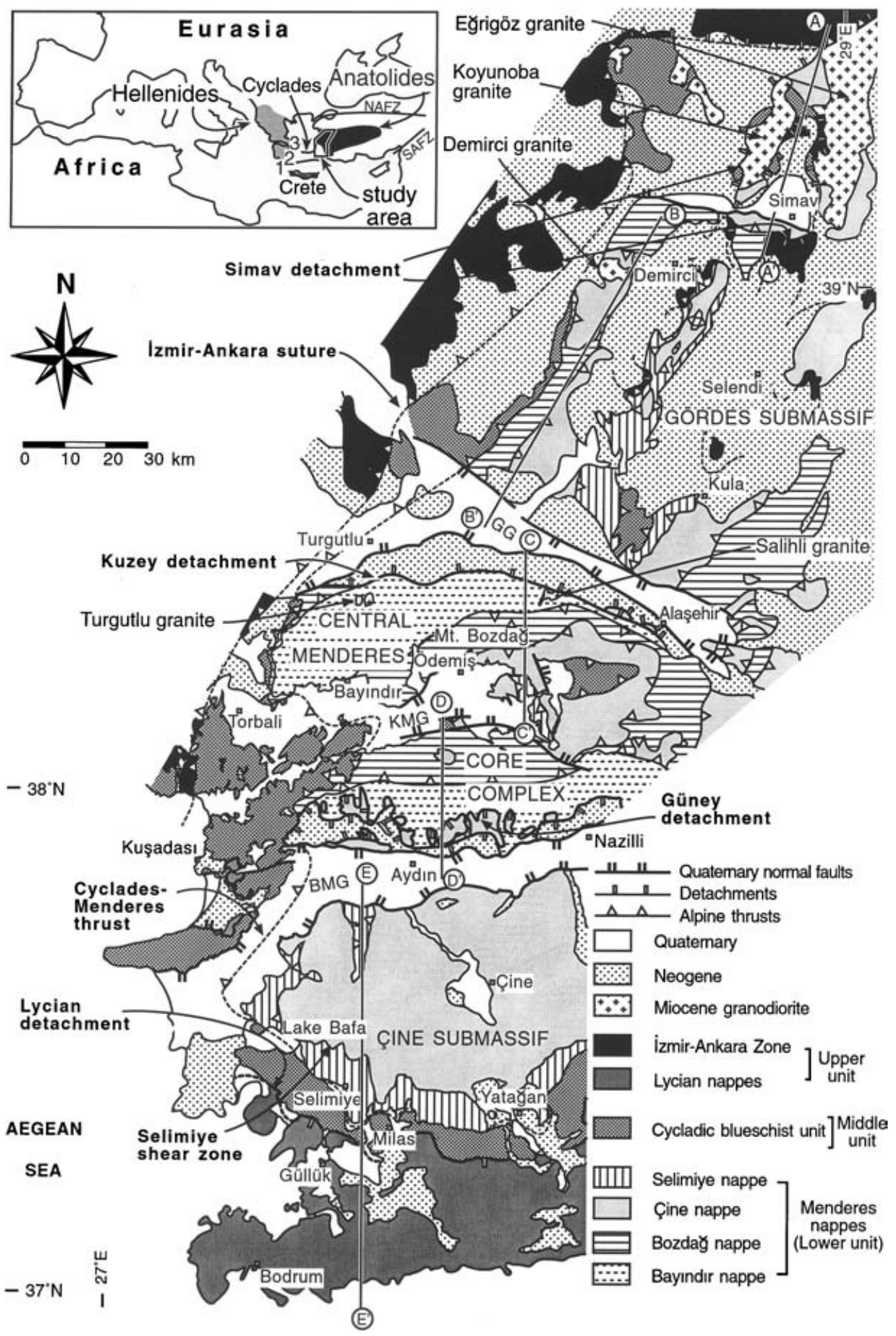

Figure 1. Simplified tectonic map of Anatolide belt in western Turkey (this region is traditionally referred to as Menderes Massif); subdivison of region into three submassifs (Gördes submassif, Ödemiş submassif, which occupies same area as the Central Menderes metamorphic core complex, and Çine submassif) follows Şengör (1987). Note that the Gördes and Çine submassifs are also referred to as outer submassifs whereas the Central Menderes metamorphic core complex represents inner submassif. Late Tertiary extension in study area is expressed by two symmetrically arranged detachment-fault systems: the Kuzey detachment in the north and Güney detachment in the south, both of which delimit the Central Menderes metamorphic core complex; also shown is Simav detachment (Işik \& Tekeli, 2001) in northern part of Gördes submassif and postulated Lycian detachment at southern tip of Çine submassif. Profile lines $\mathrm{AA}^{\prime}, \mathrm{BB}^{\prime}, \mathrm{CC}^{\prime}, \mathrm{DD}^{\prime}$ and $\mathrm{EE}^{\prime}$ refer to cross-section in Fig. 2. Abbreviations: BMG - Büyük Menderes graben, KMG - Küçük Menderes graben, GG - Gediz graben. Inset: Generalized map of the Mediterranean showing Hellenides (grey), Anatolides (black), Cyclades and Crete, location of main map and major detachment faults in Aegean; 1 - Cretan detachment, 2 - Ios and Serifos detachments, 3 - Montsuna, Vari and Mykonos detachments. Abbreviations: NAFZ - North Anatolian fault zone, SAFZ - South Anatolian fault zone. 


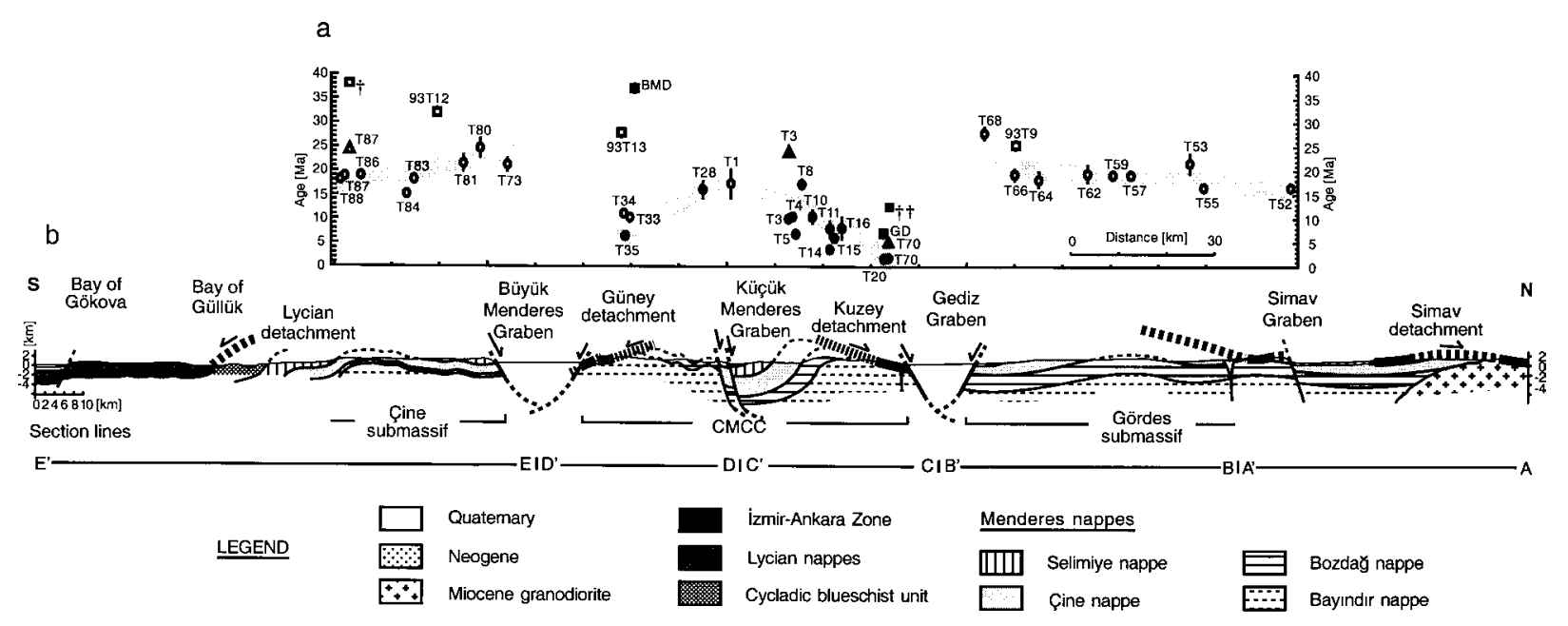

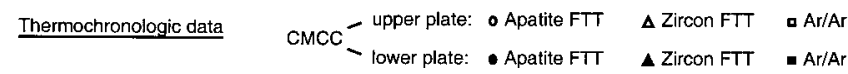

Figure 2. (a) Cooling ages in relation to position along cross-section (FTT - fission-track thermochronology); sample numbers correspond to Fig. 3 except those indicated by dagger and double daggers (samples T2, T20, 92T27, 92T54 from Hetzel \& Reischmann (1996); samples 93T18 and 93T55 from granites in footwall of Kuzey detachment from Hetzel et al. (1995a); samples GD and BMD from Lips et al. (2001)). Three ${ }^{40} \mathrm{Ar} /{ }^{39} \mathrm{Ar}$-muscovite ages from Çine nappe at southern end of profile (dagger, 93T12, 93T13) get younger with structural depth compatible with interpretation that ages are cooling ages. Note that in this diagram only samples from NNE-SSW profile (Fig. 1) are shown. (b) Schematic N-S cross-section showing architecture of nappe pile assembled during Eocene $\left(D_{A 3}\right)$ top-to-the-S nappe stacking (for profile lines refer to Fig. 1). Syncline in central part of Anatolide belt is due to large-scale folding during late phase of bivergent detachment faulting (Gessner et al. 2001a). For geometric viability, section planes are oriented parallel to mean orientation of $\mathrm{L}_{\mathrm{A} 3}$; trace of foliation is projected into section plane and used to infer geometry of sub-surface structures.

evidence for the interpretation that the Selimiye shear zone originated as a thrust that fits into the regional picture of Eocene top-to-the-S $\mathrm{D}_{\mathrm{A} 3}$ thrusts (Collins \& Robertson, 1998).

Tertiary metamorphic temperatures are difficult to constrain as Tertiary metamorphism is commonly retrogressive, overprinting pre-existing high-grade metamorphism and migmatization that has been dated at $\sim 540-550 \mathrm{Ma}$ (Dannat \& Reischmann, 1999; T. Reischmann pers. comm. 1999; Gessner et al. 2001b). Metapelite at the southern rim of the Çine submassif, which has been assigned a Devonian to Carboniferous age, contains garnet, chloritoid, muscovite, ilmenite and graphite and records Tertiary metamorphic temperatures of $500-550{ }^{\circ} \mathrm{C}$ (Whitney \& Bozkurt, 2002; Régnier et al. 2003). Triassic granite in the Bozdağ nappe south of Alaşehir also contains garnet. R. Hetzel (unpub. Ph.D. thesis, Univ. Mainz, 1995) reported garnet and biotite from the Bayindir nappe; however, the growth of these minerals might be due to contact metamorphism related to Early Miocene intrusion of the Turgutlu and Salihli granites. Metapelite in the Çine and Bozdağ nappes has no Tertiary garnet but Tertiary biotite (Ring, Willner \& Lackmann, 2001). The occurrence of biotite in the Çine and Bozdağ nappes suggests temperatures $>\sim 400^{\circ} \mathrm{C}$, while the absence of garnet suggests that Tertiary metamorphic temperatures in both nappes did not exceed $\sim 450^{\circ} \mathrm{C}$ (Bucher
\& Frey, 1994). Overall, this summary suggests that Tertiary metamorphic temperatures in the Menderes nappes were between 400 and $550^{\circ} \mathrm{C}$.

\section{2.c. Structures related to horizontal extension}

In the northern part of the Gördes submassif, Işik \& Tekeli (2001) identified the top-to-the-NNE Simav detachment (Fig. 1). The Simav detachment reactivated the Eocene Cyclades-Menderes thrust. Initial movement on the Simav detachment was synchronous with the intrusion of the Eğrigöz and Koyunoba granites, which are considerably stretched in the NNE direction. A SHRIMP-U/Pb-zircon age of $20.7 \pm 0.5 \mathrm{Ma}$ dates the intrusion of the Eğrigöz granite and thus, initial movement on the Simav detachment (Ring \& Collins, 2003). The pervasive pre-existing foliation in the footwall of the Simav detachment is subhorizontal and parallels the detachment fault. The overall geometry across the Simav detachment resembles those across detachment faults in the nearby Aegean, such as the Mykonos detachment (Lee \& Lister, 1992), the Vari detachment (Ring, Thomson \& Bröcker, 2003) or the Ios detachment (Lister \& Forster, 1996), which are thought to have originated as low-angle structures.

Contractional $\mathrm{D}_{\mathrm{A} 3}$ structures were overprinted in the Gördes submassif and in the northern Central Menderes metamorphic core complex by ductile $\mathrm{D}_{\mathrm{A} 4}$ shear bands. 
These shear bands developed in the northern Central Menderes metamorphic core complex simultaneously with the intrusion of the Early Miocene $(\sim 20 \mathrm{Ma})$ Turgutlu and Salihli granites during NNE-oriented horizontal extension (Hetzel et al. 1995a; Gessner et al. 2001c) (Fig. 3). The occurrence of andalusite in the contact aureole indicates shallow intrusion depths of $<\sim 12 \mathrm{~km}$, which suggests that the temperature of the wall rock was $<300-350^{\circ} \mathrm{C}$ during intrusion.

It has been suggested that the basal thrust of the Lycian nappes at the southern margin of the Çine submassif was reactivated in the Miocene as an extensional fault (Collins \& Robertson, 1998, 2003; Güngör \& Erdoğan, 2001), the Lycian detachment (Figs 1-3). Collins \& Robertson (1998) proposed that reactivation was due to top-to-the-E extensional faulting. Régnier et al. (2003) showed that stretching lineations indicative of tectonic transport within a narrow zone underneath the base of the Lycian nappes were passively rotated from a $\mathrm{N}-\mathrm{S}$ into an $\mathrm{E}-\mathrm{W}$ direction suggesting that the top-to-the-E shearsense indicators are rotated top-to-the-S structures. Below we will show that the cooling pattern in the southern Çine nappe is also not compatible with topto-the-E extensional faulting. Another problem is that unequivocal evidence for extensional deformation at the southern margin of the Çine submassif is lacking. The geometry of the footwall of the Lycian detachment is a large-scale S-dipping monocline that characterizes the structure of the southern margin of the Çine submassif (Fig. 2).

In the Central Menderes metamorphic core complex, in the centre of the Anatolide belt, a later phase of bivergent brittle detachment faulting is associated with the development of the Kuzey and Güney detachments and the presently active Gediz and Büyük Menderes graben (Şengör, 1987; Seyitoğlu \& Scott, 1996; Gessner et al. 2001a). Cohen et al. (1995) and subsequently Bozkurt (2000) showed that both graben formed in the Pliocene. The footwall of the top-tothe-N Kuzey detachment is a S-dipping monocline, whereas the footwall of the top-to-the-S Güney detachment is a N-dipping monocline. Both monoclines combine to form a large-scale syncline that characterizes the structure of the Central Menderes metamorphic core complex (Fig. 2). In contrast to the Simav and Lycian detachments, the Kuzey and Güney detachments did not reactivate pre-existing structures but cut across them.

This summary suggests that at least two periods of bivergent normal faulting occurred in the Anatolide belt of western Turkey. Both periods are poorly dated. The first phase may have occurred during Miocene times and affected the upper structural levels of the Menderes nappes, which are now mainly represented by the two outer submassifs. The relationship of the Early Miocene extensional $\mathrm{D}_{\mathrm{A} 4}$ shear bands in the northern Central Menderes metamorphic core complex to map-scale detachments is not known. The second phase of extensional deformation caused the development of the Central Menderes metamorphic core complex in the centre of the Anatolide belt and appears to be associated with the Pliocene graben.

\section{Sampling strategy}

For this study, orthogneiss, schist and granite samples were collected along a broadly NNE-SSW-trending transect from Simav in the north to Milas in the south across the Anatolide belt (Fig. 3). We sampled at regular intervals of $\sim 5-10 \mathrm{~km}$ to resolve the regional variation in cooling patterns across the main structural features. A causal link with denudation processes can be established by integrating the thermochronological data with additional independent geological information (Wheeler \& Butler, 1994). In the specific case of progressive unroofing of footwall rocks below a crustal-scale normal fault, asymmetric cooling is expected, resulting in decreasing apparent ages along the direction of hanging-wall transport (Brandon, Roden-Tice \& Garver, 1998; Foster \& John, 1999; Ring et al. 1999b). Apatite fission-track ages can be correlated with distance in the direction of hanging-wall motion as inferred from asymmetric kinematic indicators (Bradbury \& Nolen-Hoeksema, 1985; Foster, Miller \& Miller, 1991; Foster et al. 1993; Johnson, 1997). This feature can be used to estimate slip rates for the causative fault and has also been used to reconstruct the pre-extensional geometry of the fault system (Foster et al. 1993; Foster \& John, 1999; Gessner et al. 2001a; Ring, Thomson \& Bröcker, 2003). For these reasons, the baseline samples for this study were collected parallel to the NNE-SSW tectonic transport direction of extensional deformation (Figs 2, 3).

\section{Analytical procedures and data analysis}

Standard heavy-liquid and magnetic-separation methods were used to extract apatite, zircon and mica from the host rocks. Irradiation of the fission-track samples was carried out in the graphite reflector of the Risø reactor at Rosklide, Denmark, and monitored using Corning-glass dosimeters. Fission tracks in apatite and zircon were analysed using the external-detector and $\zeta$-calibration approach (Hurford \& Green, 1982; Hurford, 1990). Ages were calculated using the centralage method of Galbraith \& Laslett (1993), which allows for non-Poissonian variation within a population of single-grain ages belonging to an individual sample. Such variation in single-crystal ages can occur in sedimentary samples as a consequence of differing apatite provenance. The relative error or dispersion on the central age is a measure of non-Poissonian variation. Where the relative error is greater than $\sim 20 \%$, the 

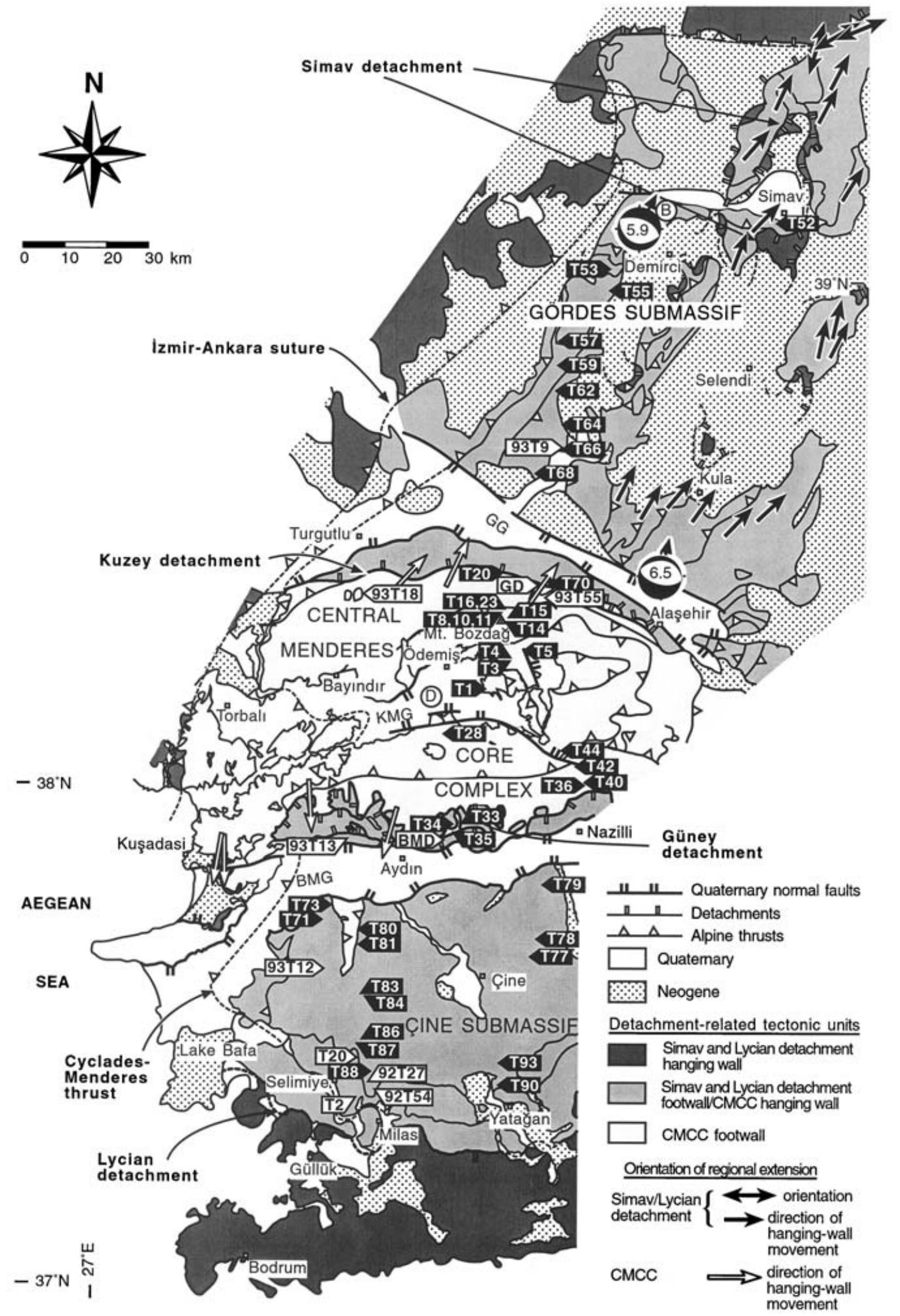

Figure 3. Map showing sample localities for apatite and zircon fission-track and ${ }^{40} \mathrm{Ar} /{ }^{39} \mathrm{Ar}$-muscovite and biotite analysis. ${ }^{40} \mathrm{Ar} /{ }^{39} \mathrm{Ar}-$ biotite data of samples $93 \mathrm{~T} 55$ and $93 \mathrm{~T} 18$ are from Hetzel et al. $(1995 a) ;{ }^{40} \mathrm{Ar} /{ }^{39} \mathrm{Ar}$-muscovite data from samples 92T27, 92T54, T2 and T20 are from Hetzel \& Reischmann (1996) and those from samples GD and BMD are from Lips et al. (2001). Also shown are extensional stretching trajectories of Late Oligocene/Early Miocene and latest Miocene/Recent phases of extensional faulting. Late Oligocene/Early Miocene top-to-the-NNE extension was ductile in northern Central Menderes metamorphic core complex and mainly brittle at Simav detachment; fault-plane solutions (lower-hemisphere focal projections, black indicates shortening quadrants), surface magnitude and slip vectors of Demirci and Alaşehir earthquakes also shown. For abbreviations, see Fig. 1. 
sample is also likely to fail the standard $\chi^{2}$ test and the spread in single-grain ages is often attributable to the presence of different age populations.

Some fission-track ages from the Anatolide belt do not have single-age spreads consistent with a singleage population when assessed using the relative error and the $\chi^{2}$ test. We think that the scatter in single ages is due to errors of commission and omission that resulted from the frequent presence of track-like crystal defects. Although possibly small in number, the misidentification of very short tracks and spurious etch pits has a significant effect because true spontaneous track numbers are low. The large number of crystals used and the likelihood that these two types of error are equally common means that they will tend to cancel out when the central age is calculated but produce a large single-grain-age spread. This conclusion is supported by the age occurrence of closely spaced samples, some of which fail the single-population tests while their neighbours do not.

Track-length data were obtained using a drawing tube and digitizing tablet. Typically, 100 track-length measurements were made for determining the mean track length and its standard deviation for track-length modelling and the construction of a thermal history. Fifteen of the 44 apatites analysed matched these criteria, while for 19 samples we obtained less than 100 track-length measurements. For 10 samples we did not obtain any track-length data.

The procedures for multi-grain furnace ${ }^{40} \mathrm{Ar} /{ }^{39} \mathrm{Ar}$ analysis of mica from weakly deformed orthogneiss are detailed in Hetzel et al. (1995a) and Hetzel \& Reischmann (1996). The argon isotopic composition was measured in static mode on a MAT GD-150 mass spectrometer at the Max-Planck Institut für Chemie in Mainz, Germany. The ${ }^{40} \mathrm{Ar} /{ }^{39} \mathrm{Ar}-$ mica (Fig. 4) and the zircon fission-track ages have been used together with the apatite fission-track data (Table 1) to construct cooling curves.

Thermochronological data have been interpreted in terms of a temperature history by using a nominal 'closure temperature' in conjunction with the mineral age as a point on a time-temperature history plot (e.g. Hurford, 1986). The following closure temperatures have been used in this study: $\sim 110 \pm 10^{\circ} \mathrm{C}$ for fluorapatite (Gleadow \& Duddy, 1981) and $240-280^{\circ} \mathrm{C}$ for zircon (Tagami \& Dumitru, 1996). The isotopic closure temperatures for Ar in biotite and muscovite are controversial. As shown by Villa \& Puxeddu (1994) in a study in the Lardello geothermal field, experimentally derived diffusion coefficients were about three times greater than those derived from laboratory experiments suggesting that the closure temperature for Ar diffusion in mica is higher than that derived from diffusion experiments. We assume closure temperatures of $\sim 350^{\circ} \mathrm{C}$ for biotite and $400-450{ }^{\circ} \mathrm{C}$ for muscovite, which are about $\pm 50^{\circ} \mathrm{C}$ higher than the widely accepted values. However, we emphasize that our choice does not affect the general conclusions of this study in any significant way.

More detailed thermal history information can be obtained from apatite fission-track data using a quantitative approach, based on empirically calibrated algorithms (e.g. Laslett et al. 1987) and a search algorithm to examine a wide range of time-temperature space for acceptable thermal history models (e.g. Gallagher, 1995). We used the modelling method of Gallagher (1995) and the annealing model for Durango apatite of Laslett et al. (1987) to obtain thermal histories. To define an acceptable thermal history, we used a likelihood function (Gallagher, 1995), which is a measure of the probability that a given thermal history would have produced the observed data. Acceptable models are found by a stochastic search, using a genetic algorithm, of a model timetemperature space. In this method, the sampling of thermal histories is progressively biased toward those histories which maximize the likelihood of obtaining the observed data. In this study, a typical modelling run considered $\sim 10000$ thermal histories defined by four to ten time-temperature points for a given sample location. We varied the model time-temperature input parameters until the predicted age and mean length of the modelling run was within $5 \%$ of the measured age and track length.

Regional variations in the thermal histories of all samples are readily visualized through contouring the site-specific temperature estimates at given times. One way of displaying the cooling history of an orogen is to convert thermal history information into maps for different time intervals (Gleadow et al. 1999) (Fig. 5). To construct such maps, we used the 15 apatites for which we obtained 100 or more tracklength measurements, and those results were used to help interpret the 19 less well-constrained samples.

\section{Thermochronological data}

\section{5.a. Results}

Forty-four apatite fission-track analyses were completed whose central ages vary between $27.9 \pm 1.2 \mathrm{Ma}$ and $1.8 \pm 0.6 \mathrm{Ma}$. In addition to the apatite results, four zircon analyses have central ages between $27.9 \pm 1.5 \mathrm{Ma}$ and $5.2 \pm 1.1 \mathrm{Ma}$ (Table 1). Ages from all samples are much younger than the crystallization or (pre-metamorphic) sedimentation age of the rocks from which they were obtained. Therefore, the fissiontrack analyses (central-age and track-length data) are interpreted as yielding cooling history information. The ${ }^{40} \mathrm{Ar} /{ }^{39} \mathrm{Ar}$-muscovite-isochron ages range between $31.6 \pm 1.2 \mathrm{Ma}$ and $24.7 \pm 1.2 \mathrm{Ma}$ (Fig. 4). All sample localities and additional samples for ${ }^{40} \mathrm{Ar} /{ }^{39} \mathrm{Ar}$ dating reported in Hetzel et al. (1995a), Hetzel \& Reischmann (1996) and Lips et al. (2001) are shown in Fig. 3. 

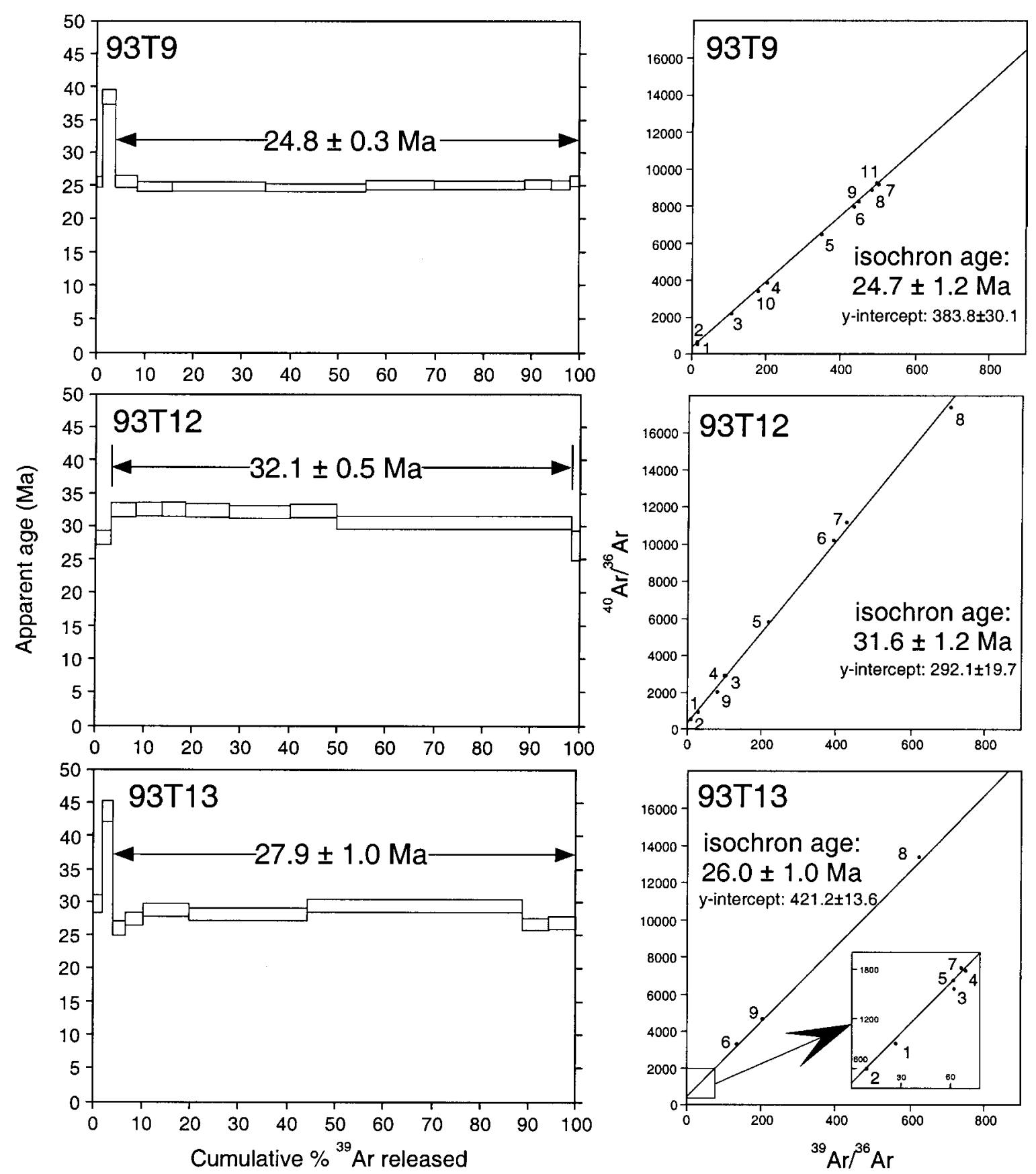

Figure 4. ${ }^{40} \mathrm{Ar} /{ }^{39} \mathrm{Ar}-\mathrm{muscovite}$ data from samples 93T9, $93 \mathrm{~T} 12$ and $92 \mathrm{~T} 13$ (for localities refer to Fig. 3). Shown are muscovite release spectra on left-hand side and isotope-correlation diagrams on right-hand side of figure; release steps, which have been used for calculation of plateau ages are indicated by arrows. Sequence of heating steps in each isotope-correlation diagram is shown by numbers.

\section{5.b. Interpretation of apatite fission-track data}

The results of thermal history modelling of the apatite fission-track data are presented as a series of contoured time-temperature slices, advancing in nine steps from Early Miocene times to the present (Fig. 6). Note that the contouring is strongly controlled by the distribution of sample points (Fig. 3) and the discussion below should be considered accordingly. Prior to $\sim 30 \mathrm{Ma}$, temperatures for all samples are greater than $\sim 130^{\circ} \mathrm{C}$.
The apatite fission-track data alone do not yield any information about the thermal evolution of the Anatolide belt prior to this time. The main features of these maps are listed below.

$28 \mathrm{Ma}$ : The first recorded cooling in the belt to temperatures within the apatite partial-annealing zone $\left(\sim 110-60^{\circ} \mathrm{C}\right)$ (Fitzgerald et al. 1995) occurred on the southern margin of the Gördes submassif. Initial cooling is based entirely on the age of $27.9 \pm 1.2 \mathrm{Ma}$ of sample T68. 
Table 1. Fission-track (FT) data for apatite and zircon and sample localities

\begin{tabular}{|c|c|c|c|c|c|c|c|c|c|c|}
\hline Sample & $\begin{array}{l}\text { Location } \\
\left(\text { Lat }^{\circ} \mathrm{N} /\right. \\
\left.\text { Long }^{\circ} \mathrm{E}\right)\end{array}$ & $\begin{array}{l}\text { Elevation } \\
\text { (m) }\end{array}$ & $\begin{array}{l}\text { Mineral } \\
\text { and no. of } \\
\text { crystals }\end{array}$ & $\begin{array}{c}\text { Spontaneous } \\
\rho_{\mathrm{s}} \\
\left(\mathrm{N}_{\mathrm{s}}\right)\end{array}$ & $\begin{array}{c}\text { Induced } \\
\rho_{\mathrm{i}} \\
\left(\mathrm{N}_{\mathrm{i}}\right)\end{array}$ & $\begin{array}{c}\text { Dosimeter } \\
\rho_{\mathrm{d}} \\
\left(\mathrm{N}_{\mathrm{d}}\right)\end{array}$ & $\begin{array}{c}\text { Central } \\
\text { FT age } \\
(\mathrm{Ma})( \pm 1 \sigma)\end{array}$ & $\begin{array}{l}\text { Relative } \\
\text { error } \\
\%\end{array}$ & $\begin{array}{l}\text { MCTL } \ddagger(\mu \mathrm{m}) \\
\text { and no. of } \\
\text { tracks }\end{array}$ & $\begin{array}{l}\text { S.D. } \\
1 \sigma\end{array}$ \\
\hline $\mathrm{T} 1$ & $\begin{array}{l}38.18317 \\
28.06286\end{array}$ & 150 & Apatite (25) & $\begin{array}{c}0.035 \\
(51)\end{array}$ & $\begin{array}{l}0.038 \\
(552)\end{array}$ & $\begin{array}{l}0.331 \\
(9219)\end{array}$ & $22.15 \pm 3.26$ & 3.17 & $\begin{array}{c}13.70 \pm 0.31 \\
(14)\end{array}$ & 0.75 \\
\hline $\mathrm{T} 3$ & $\begin{array}{l}38.24981 \\
28.12811\end{array}$ & 700 & Apatite (25) & $\begin{array}{l}0.121 \\
(333)\end{array}$ & $\begin{array}{c}0.288 \\
(7952)\end{array}$ & $\begin{array}{c}0.331 \\
(9219)\end{array}$ & $10.05 \pm 0.57$ & 0.75 & $\begin{array}{c}14.23 \pm 0.16 \\
(63)\end{array}$ & 1.28 \\
\hline $\mathrm{T} 3$ & $\begin{array}{l}38.24981 \\
28.12811\end{array}$ & 700 & Zircon (20) & $\begin{array}{l}3.024 \\
(2846)\end{array}$ & $\begin{array}{c}0.340 \\
(3198)\end{array}$ & $\begin{array}{c}0.039 \\
(2729)\end{array}$ & $24.20 \pm 1.12$ & 14.62 & N.D. & N.A. \\
\hline $\mathrm{T} 4$ & $\begin{array}{l}38.24514 \\
28.10472\end{array}$ & 420 & Apatite (16) & $\begin{array}{l}0.318 \\
(504)\end{array}$ & $\begin{array}{c}0.718 \\
(11372)\end{array}$ & $\begin{array}{l}0.133 \\
(9219)\end{array}$ & $10.64 \pm 0.64$ & 13.41 & $\begin{array}{l}13.92 \pm 0.15 \\
\quad(100)\end{array}$ & 1.45 \\
\hline T5 & $\begin{array}{l}38.24755 \\
28.09378\end{array}$ & 380 & Apatite (22) & $\begin{array}{l}0.052 \\
(158)\end{array}$ & $\begin{array}{c}0.153 \\
(4640)\end{array}$ & $\begin{array}{c}0.113 \\
(7801)\end{array}$ & $6.92 \pm 0.56$ & 0.00 & $\begin{array}{c}14.34 \pm 0.23 \\
(33)\end{array}$ & 1.28 \\
\hline $\mathrm{T} 8$ & $\begin{array}{l}38.16289 \\
28.03477\end{array}$ & 830 & Apatite (20) & $\begin{array}{l}0.235 \\
(280)\end{array}$ & $\begin{array}{c}0.312 \\
(3725)\end{array}$ & $\begin{array}{c}0.126 \\
(5223)\end{array}$ & $17.03 \pm 1.08$ & 0.01 & $\begin{array}{l}14.14 \pm 0.19 \\
(100)\end{array}$ & 1.84 \\
\hline $\mathrm{T} 10$ & $\begin{array}{l}38.17449 \\
28.03162\end{array}$ & 1180 & Apatite (20) & $\begin{array}{l}0.027 \\
(58)\end{array}$ & $\begin{array}{c}0.087 \\
(1850)\end{array}$ & $\begin{array}{c}0.185 \\
(12840)\end{array}$ & $10.48 \pm 1.40$ & 0.02 & N.D. & N.A. \\
\hline $\mathrm{T} 11$ & $\begin{array}{l}38.20162 \\
28.04291\end{array}$ & 1200 & Apatite (19) & $\begin{array}{c}0.011 \\
(21)\end{array}$ & $\begin{array}{l}0.046 \\
(888)\end{array}$ & $\begin{array}{c}0.185 \\
(12840)\end{array}$ & $7.90 \pm 1.75$ & 0.00 & N.D. & N.A. \\
\hline $\mathrm{T} 14$ & $\begin{array}{l}38.19179 \\
28.06113\end{array}$ & 2150 & Apatite (30) & $\begin{array}{l}0.025 \\
(38)\end{array}$ & $\begin{array}{c}0.161 \\
(2446)\end{array}$ & $\begin{array}{l}0.1331 \\
(9219)\end{array}$ & $3.73 \pm 0.61$ & 0.09 & $\begin{array}{c}14.19 \pm 0.28 \\
(23)\end{array}$ & 1.33 \\
\hline $\mathrm{T} 15$ & $\begin{array}{l}38.19179 \\
28.06182\end{array}$ & 1920 & Apatite (25) & $\begin{array}{l}1.037 \\
(55)\end{array}$ & $\begin{array}{c}0.146 \\
(2171)\end{array}$ & $\begin{array}{l}0.1331 \\
(9219)\end{array}$ & $6.08 \pm 0.83$ & 0.63 & $\begin{array}{c}14.08 \pm 0.68 \\
(18)\end{array}$ & 2.79 \\
\hline T16 & $\begin{array}{l}38.20194 \\
28.06129\end{array}$ & 1700 & Apatite (50) & $\begin{array}{c}0.005 \\
(12)\end{array}$ & $\begin{array}{l}0.014 \\
(356)\end{array}$ & $\begin{array}{l}0.1331 \\
(9219)\end{array}$ & $8.74 \pm 2.67$ & 59.94 & N.D. & N.A. \\
\hline $\mathrm{T} 20$ & $\begin{array}{l}38.26466 \\
28.04200\end{array}$ & 370 & Apatite (30) & $\begin{array}{l}0.005 \\
(8)\end{array}$ & $\begin{array}{c}0.067 \\
(1035)\end{array}$ & $\begin{array}{c}0.126 \\
(5223)\end{array}$ & $1.75 \pm 0.62$ & 0.10 & N.D. & N.A. \\
\hline $\mathrm{T} 23$ & $\begin{array}{l}38.22404 \\
27.40393\end{array}$ & 740 & Apatite (10) & $\begin{array}{l}0.493 \\
(384)\end{array}$ & $\begin{array}{c}1.106 \\
(8606)\end{array}$ & $\begin{array}{c}0.126 \\
(5223)\end{array}$ & $10.12 \pm 0.55$ & 0.51 & $\begin{array}{l}14.10 \pm 0.16 \\
\text { (71) }\end{array}$ & 1.35 \\
\hline T28 & $\begin{array}{l}38.05474 \\
27.53518\end{array}$ & 160 & Apatite (20) & $\begin{array}{l}0.111 \\
(151)\end{array}$ & $\begin{array}{c}0.245 \\
(3329)\end{array}$ & $\begin{array}{c}0.185 \\
(12840)\end{array}$ & $16.11 \pm 1.74$ & 24.83 & $\begin{array}{c}14.03 \pm 0.23 \\
(36)\end{array}$ & 1.36 \\
\hline T33 & $\begin{array}{l}37.57289 \\
28.01457\end{array}$ & 660 & Apatite (5) & $\begin{array}{l}0.555 \\
(142)\end{array}$ & $\begin{array}{c}1.119 \\
(2861)\end{array}$ & $\begin{array}{l}0.115 \\
(7926)\end{array}$ & $10.25 \pm 0.89$ & 0.11 & $\begin{array}{c}11.87 \pm 0.14 \\
(70)\end{array}$ & 1.15 \\
\hline T34 & $\begin{array}{l}37.56113 \\
28.01196\end{array}$ & 310 & Apatite (10) & $\begin{array}{l}0.616 \\
(474)\end{array}$ & $\begin{array}{c}1.164 \\
(8965)\end{array}$ & $\begin{array}{l}0.115 \\
(7926)\end{array}$ & $11.12 \pm 0.64$ & 8.96 & $\begin{array}{c}11.87 \pm 0.14 \\
(100)\end{array}$ & 1.39 \\
\hline T35 & $\begin{array}{l}37.55425 \\
28.01400\end{array}$ & 180 & Apatite (21) & $\begin{array}{l}0.118 \\
(200)\end{array}$ & $\begin{array}{c}0.375 \\
(6389)\end{array}$ & $\begin{array}{c}0.115 \\
(7926)\end{array}$ & $6.46 \pm 0.49$ & 7.08 & $\begin{array}{c}11.48 \pm 0.29 \\
(50)\end{array}$ & 2.05 \\
\hline T36 & $\begin{array}{l}37.57244 \\
28.17586\end{array}$ & 450 & Apatite (20) & $\begin{array}{l}0.132 \\
(276)\end{array}$ & $\begin{array}{c}0.178 \\
(3735)\end{array}$ & $\begin{array}{c}0.120 \\
(4984)\end{array}$ & $15.98 \pm 1.05$ & 5.02 & $\begin{array}{c}13.23 \pm 0.42 \\
(32)\end{array}$ & 2.33 \\
\hline $\mathrm{T} 40$ & $\begin{array}{l}38.02211 \\
28.17405\end{array}$ & 930 & Apatite (10) & $\begin{array}{c}0.267 \\
(83)\end{array}$ & $\begin{array}{c}0.591 \\
(1839)\end{array}$ & $\begin{array}{c}0.185 \\
(12840)\end{array}$ & $16.35 \pm 3.42$ & 52.60 & $\begin{array}{c}15.70 \pm 0.54 \\
(6)\end{array}$ & 1.22 \\
\hline $\mathrm{T} 42$ & $\begin{array}{l}38.02543 \\
28.16330\end{array}$ & 750 & Apatite (10) & $\begin{array}{c}0.122 \\
(58)\end{array}$ & $\begin{array}{c}0.277 \\
(1315)\end{array}$ & $\begin{array}{c}0.185 \\
(12840)\end{array}$ & $14.16 \pm 2.73$ & 28.33 & $\begin{array}{c}14.03 \pm 0.23 \\
(36)\end{array}$ & 1.36 \\
\hline $\mathrm{T} 44$ & $\begin{array}{l}38.03553 \\
28.14225\end{array}$ & 430 & Apatite (20) & $\begin{array}{l}0.253 \\
(538)\end{array}$ & $\begin{array}{c}0.649 \\
(13782)\end{array}$ & $\begin{array}{c}0.185 \\
(12840)\end{array}$ & $13.03 \pm 0.60$ & 4.67 & $\begin{array}{l}14.47 \pm 0.15 \\
\quad(100)\end{array}$ & 1.49 \\
\hline T52 & $\begin{array}{l}39.04529 \\
28.55327\end{array}$ & 1550 & Apatite (11) & $\begin{array}{l}0.502 \\
(617)\end{array}$ & $\begin{array}{c}1.017 \\
(12495)\end{array}$ & $\begin{array}{c}0.185 \\
(12840)\end{array}$ & $16.53 \pm 0.72$ & 3.24 & $\begin{array}{l}14.55 \pm 0.11 \\
(106)\end{array}$ & 1.14 \\
\hline T53 & $\begin{array}{l}38.57307 \\
28.30374\end{array}$ & 520 & Apatite (25) & $\begin{array}{l}0.049 \\
(105)\end{array}$ & $\begin{array}{c}0.075 \\
(1632)\end{array}$ & $\begin{array}{c}0.185 \\
(12840)\end{array}$ & $21.48 \pm 2.17$ & 1.28 & N.D. & N.A. \\
\hline T55 & $\begin{array}{l}39.00096 \\
28.26199\end{array}$ & 750 & Apatite (18) & $\begin{array}{l}0.554 \\
(671)\end{array}$ & $\begin{array}{c}1.158 \\
(14042)\end{array}$ & $\begin{array}{c}0.185 \\
(12840)\end{array}$ & $16.05 \pm 1.08$ & 21.60 & $\begin{array}{l}14.22 \pm 0.13 \\
\quad(100)\end{array}$ & 1.28 \\
\hline T57 & $\begin{array}{l}38.53052 \\
28.19437\end{array}$ & 700 & Apatite (15) & $\begin{array}{l}0.552 \\
(592)\end{array}$ & $\begin{array}{c}0.643 \\
(6898)\end{array}$ & $\begin{array}{c}0.120 \\
(4984)\end{array}$ & $18.58 \pm 0.85$ & 2.93 & $\begin{array}{l}14.23 \pm 0.12 \\
\quad(100)\end{array}$ & 1.16 \\
\hline T59 & $\begin{array}{l}38.50520 \\
28.19433\end{array}$ & 790 & Apatite (10) & $\begin{array}{c}1.137 \\
(1185)\end{array}$ & $\begin{array}{c}1.323 \\
(13795)\end{array}$ & $\begin{array}{c}0.120 \\
(4984)\end{array}$ & $18.58 \pm 0.62$ & 0.33 & $\begin{array}{c}14.47 \pm 0.10 \\
(100)\end{array}$ & 1.00 \\
\hline T62 & $\begin{array}{l}38.47039 \\
28.20225\end{array}$ & 600 & Apatite (20) & $\begin{array}{l}0.084 \\
(124)\end{array}$ & $\begin{array}{c}0.096 \\
(1415)\end{array}$ & $\begin{array}{c}0.120 \\
(4984)\end{array}$ & $18.98 \pm 1.81$ & 4.30 & $\begin{array}{c}14.59 \pm 0.21 \\
(24)\end{array}$ & 0.99 \\
\hline T64 & $\begin{array}{l}38.41336 \\
28.19438\end{array}$ & 340 & Apatite (20) & $\begin{array}{l}0.144 \\
(321)\end{array}$ & $\begin{array}{c}0.197 \\
(4383)\end{array}$ & $\begin{array}{c}0.126 \\
(5223)\end{array}$ & $18.24 \pm 1.61$ & 24.44 & $\begin{array}{c}14.60 \pm 0.22 \\
(43)\end{array}$ & 1.41 \\
\hline T66 & $\begin{array}{l}38.38253 \\
28.20245\end{array}$ & 280 & Apatite (20) & $\begin{array}{l}0.163 \\
(328)\end{array}$ & $\begin{array}{c}0.183 \\
(3686)\end{array}$ & $\begin{array}{c}0.120 \\
(4984)\end{array}$ & $19.24 \pm 1.17$ & 4.63 & $\begin{array}{c}14.58 \pm 0.22 \\
(31)\end{array}$ & 1.20 \\
\hline T68 & $\begin{array}{l}38.35264 \\
28.17245\end{array}$ & 220 & Apatite (10) & $\begin{array}{l}1.164 \\
(997)\end{array}$ & $\begin{array}{c}0.911 \\
(7800)\end{array}$ & $\begin{array}{c}0.120 \\
(4984)\end{array}$ & $27.85 \pm 1.22$ & 7.06 & $\begin{array}{c}14.32 \pm 0.12 \\
(101)\end{array}$ & 1.16 \\
\hline $\mathrm{T} 70$ & $\begin{array}{l}38.24295 \\
28.14192\end{array}$ & 530 & Apatite (40) & $\begin{array}{c}0.013 \\
(27)\end{array}$ & $\begin{array}{c}0.158 \\
(3270)\end{array}$ & $\begin{array}{c}0.126 \\
(5223)\end{array}$ & $1.87 \pm 0.36$ & 2.60 & N.D. & N.A. \\
\hline $\mathrm{T} 70$ & $\begin{array}{l}38.24295 \\
28.14192\end{array}$ & 530 & Zircon (8) & $\begin{array}{l}1.087 \\
(742)\end{array}$ & $\begin{array}{c}0.564 \\
(3849)\end{array}$ & $\begin{array}{c}0.039 \\
(2729)\end{array}$ & $5.19 \pm 0.28$ & 8.29 & N.D. & N.A. \\
\hline
\end{tabular}


Table 1 (cont.)

\begin{tabular}{|c|c|c|c|c|c|c|c|c|c|c|}
\hline Sample & $\begin{array}{l}\text { Location } \\
\left(\text { Lat }^{\circ} \mathrm{N} /\right. \\
\left.\text { Long }^{\circ} \mathrm{E}\right)\end{array}$ & $\begin{array}{l}\text { Elevation } \\
\text { (m) }\end{array}$ & $\begin{array}{l}\text { Mineral } \\
\text { and no. of } \\
\text { crystals }\end{array}$ & $\begin{array}{c}\text { Spontaneous } \\
\rho_{\mathrm{s}} \\
\left(\mathrm{N}_{\mathrm{s}}\right)\end{array}$ & $\begin{array}{c}\text { Induced } \\
\rho_{\mathrm{i}} \\
\left(\mathrm{N}_{\mathrm{i}}\right)\end{array}$ & $\begin{array}{c}\text { Dosimeter } \\
\rho_{\mathrm{d}} \\
\left(\mathrm{N}_{\mathrm{d}}\right)\end{array}$ & $\begin{array}{c}\text { Central } \\
\text { FT age } \\
(\mathrm{Ma})( \pm 1 \sigma)\end{array}$ & $\begin{array}{l}\text { Relative } \\
\text { error } \\
\%\end{array}$ & $\begin{array}{l}\text { MCTL } \ddagger(\mu \mathrm{m}) \\
\text { and no. of } \\
\text { tracks }\end{array}$ & $\begin{array}{l}\text { S.D. } \\
1 \sigma\end{array}$ \\
\hline $\mathrm{T} 71$ & $\begin{array}{l}37.49338 \\
28.21282\end{array}$ & 90 & Apatite (20) & $\begin{array}{l}0.282 \\
(646)\end{array}$ & $\begin{array}{c}0.298 \\
(6838)\end{array}$ & $\begin{array}{c}0.133 \\
(9219)\end{array}$ & $22.57 \pm 1.10$ & 9.47 & $\begin{array}{c}14.00 \pm 0.12 \\
(102)\end{array}$ & 1.24 \\
\hline $\mathrm{T} 73$ & $\begin{array}{l}37.44209 \\
28.21146\end{array}$ & 110 & Apatite (15) & $\begin{array}{c}0.946 \\
(1210)\end{array}$ & $\begin{array}{c}0.117 \\
(11931)\end{array}$ & $\begin{array}{c}0.117 \\
(8098)\end{array}$ & $21.66 \pm 1.16$ & 15.88 & $\begin{array}{c}14.42 \pm 0.08 \\
(100)\end{array}$ & 0.82 \\
\hline T77 & $\begin{array}{l}37.40044 \\
28.16149\end{array}$ & 1110 & Apatite (20) & $\begin{array}{l}0.178 \\
(224)\end{array}$ & $\begin{array}{c}0.164 \\
(2064)\end{array}$ & $\begin{array}{c}0.117 \\
(8098)\end{array}$ & $22.86 \pm 1.63$ & 0.20 & $\begin{array}{c}14.87 \pm 0.18 \\
(62)\end{array}$ & 1.44 \\
\hline T78 & $\begin{array}{l}37.40286 \\
28.15534\end{array}$ & 1270 & Apatite (30) & $\begin{array}{l}0.550 \\
(131)\end{array}$ & $\begin{array}{c}0.559 \\
(1332)\end{array}$ & $\begin{array}{c}0.117 \\
(8098)\end{array}$ & $21.04 \pm 2.19$ & 23.79 & $\begin{array}{c}14.82 \pm 0.19 \\
\quad(36)\end{array}$ & 1.12 \\
\hline T79 & $\begin{array}{l}37.45566 \\
27.43026\end{array}$ & 30 & Apatite (20) & $\begin{array}{l}0.143 \\
(225)\end{array}$ & $\begin{array}{l}0.141 \\
(2224)\end{array}$ & $\begin{array}{c}0.117 \\
(8098)\end{array}$ & $21.31 \pm 1.80$ & 0.51 & $\begin{array}{c}14.40 \pm 0.22 \\
\quad(41)\end{array}$ & 1.36 \\
\hline T80 & $\begin{array}{l}37.43287 \\
27.40582\end{array}$ & 570 & Apatite (21) & $\begin{array}{l}0.066 \\
(155)\end{array}$ & $\begin{array}{c}0.055 \\
(1294)\end{array}$ & $\begin{array}{c}0.115 \\
(7926)\end{array}$ & $24.68 \pm 2.19$ & 10.54 & N.D. & N.A. \\
\hline T81 & $\begin{array}{l}37.40532 \\
27.41203\end{array}$ & 750 & Apatite (19) & $\begin{array}{l}0.071 \\
(134)\end{array}$ & $\begin{array}{c}0.068 \\
(1285)\end{array}$ & $\begin{array}{c}0.115 \\
(7926)\end{array}$ & $21.52 \pm 1.98$ & 10.54 & N.D. & N.A. \\
\hline $\mathrm{T} 83$ & $\begin{array}{l}37.35148 \\
27.42026\end{array}$ & 550 & Apatite (25) & $\begin{array}{l}0.193 \\
(569)\end{array}$ & $\begin{array}{c}0.218 \\
(6419)\end{array}$ & $\begin{array}{c}0.115 \\
(7926)\end{array}$ & $18.23 \pm 0.96$ & 10.54 & $\begin{array}{c}14.46 \pm 0.12 \\
(100)\end{array}$ & 1.17 \\
\hline T84 & $\begin{array}{l}37.33585 \\
27.43101\end{array}$ & 550 & Apatite (20) & $\begin{array}{l}0.766 \\
(212)\end{array}$ & $\begin{array}{c}0.105 \\
(2895)\end{array}$ & $\begin{array}{c}0.115 \\
(7926)\end{array}$ & $15.12 \pm 1.09$ & 0.05 & $\begin{array}{c}14.59 \pm 0.13 \\
(100)\end{array}$ & 1.31 \\
\hline T86 & $\begin{array}{l}37.29484 \\
27.43002\end{array}$ & 610 & Apatite (20) & $\begin{array}{l}0.239 \\
(599)\end{array}$ & $\begin{array}{c}0.256 \\
(6427)\end{array}$ & $\begin{array}{c}0.115 \\
(7926)\end{array}$ & $19.06 \pm 1.11$ & 14.38 & $\begin{array}{c}14.77 \pm 0.14 \\
(100)\end{array}$ & 1.37 \\
\hline T87 & $\begin{array}{l}37.28443 \\
27.42345\end{array}$ & 860 & Apatite (25) & $\begin{array}{l}0.213 \\
(477)\end{array}$ & $\begin{array}{c}0.245 \\
(5480)\end{array}$ & $\begin{array}{c}0.115 \\
(7926)\end{array}$ & $18.20 \pm 1.15$ & 18.61 & $\begin{array}{c}14.34 \pm 0.10 \\
(89)\end{array}$ & 0.98 \\
\hline T87 & $\begin{array}{l}37.28443 \\
27.42345\end{array}$ & 860 & Zircon (15) & $\begin{array}{l}2.900 \\
(2846)\end{array}$ & $\begin{array}{c}0.316 \\
(2520)\end{array}$ & $\begin{array}{c}0.039 \\
(2729)\end{array}$ & $24.69 \pm 0.90$ & 4.48 & N.D. & N.A. \\
\hline T88 & $\begin{array}{l}37.26425 \\
27.41234\end{array}$ & 480 & Apatite (25) & $\begin{array}{l}0.193 \\
(569)\end{array}$ & $\begin{array}{c}0.218 \\
(6419)\end{array}$ & $\begin{array}{c}0.115 \\
(7926)\end{array}$ & $18.23 \pm 0.96$ & 10.54 & N.D. & N.A. \\
\hline T90 & $\begin{array}{l}37.24559 \\
28.09262\end{array}$ & 380 & Apatite (20) & $\begin{array}{l}0.105 \\
(239)\end{array}$ & $\begin{array}{c}0.137 \\
(3103)\end{array}$ & $\begin{array}{c}0.117 \\
(8098)\end{array}$ & $15.82 \pm 1.25$ & 13.69 & N.D. & N.A. \\
\hline T90 & $\begin{array}{l}37.24559 \\
28.09262\end{array}$ & 380 & Zircon (10) & $\begin{array}{l}2.123 \\
(779)\end{array}$ & $\begin{array}{l}0.205 \\
(752)\end{array}$ & $\begin{array}{c}0.039 \\
(2729)\end{array}$ & $27.88 \pm 1.52$ & 0.00 & N.D. & N.A. \\
\hline T93 & $\begin{array}{l}37.39591 \\
28.06173\end{array}$ & 130 & Apatite (20) & $\begin{array}{l}0.125 \\
(156)\end{array}$ & $\begin{array}{c}0.124 \\
(1548)\end{array}$ & $\begin{array}{c}0.117 \\
(8098)\end{array}$ & $21.23 \pm 1.80$ & 0.01 & $\begin{array}{c}13.70 \pm 0.17 \\
(100)\end{array}$ & 1.65 \\
\hline
\end{tabular}

Dosimeter track densities $\left(\rho_{\mathrm{d}}\right)$ are $\cdot 10^{6} \mathrm{tr}_{\mathrm{cm}}^{-2}$; other track densities $\left(\rho_{\mathrm{s}}, \rho_{\mathrm{i}}\right)$ are $\cdot 10^{7} \operatorname{tr}^{-2}$, except apatite where $\left(\rho_{\mathrm{s}}\right)$ is $\cdot 10^{5} \operatorname{tr} \mathrm{cm}{ }^{-2}$; number of tracks counted shown in brackets.

Analyses are by external-detector method using 0.5 for $4 \pi / 2 \pi$ geometry-correction factor.

Zircon ages calculated using dosimeter glass $\mathrm{CN} 2$ with $\zeta_{\mathrm{CN} 2}=137 \pm 2.14$; apatite ages calculated using dosimeter glass $\mathrm{CN} 5$ with $\zeta_{\mathrm{CN} 5}=$ $361 \pm 6$.

S.D. = standard deviation; MCTL $\ddagger=$ mean confined track length; N.D. = not determined; N.A. = not applicable.

$25 \mathrm{Ma}$ : Cooling into the uppermost apatite partialannealing zone occurred in the northern Çine submassif. At the same time cooling to temperatures in the lower apatite partial-annealing zone occurred in the southern Gördes submassif.

$22 \mathrm{Ma}$ : Cooling started in a down-faulted block in the Küçük Menderes graben in the central part of the Central Menderes metamorphic core complex. Further cooling occurred in the Çine submassif; the locus of cooling appears to be in the southeastern sector of the submassif. Note that in the southern Gördes submassif the cooling pattern is still controlled by sample T68; all other samples are still above $\sim 130^{\circ} \mathrm{C}$.

$20 \mathrm{Ma}$ : In the Gördes submassif, cooling started to progress northward. The samples in the Gördes submassif are not evenly distributed but instead lie on one single NNE-SSW transect, and the limited E-W distribution of sample points restricts the interpretation of the data in this orientation. However, it seems reasonable to speculate that cooling in the entire Gördes submassif propagated northward. Cooling in the Çine submassif continued and, in general, it seems that cooling progressed in a southerly direction.

$18 \mathrm{Ma}$ : By this time much of the southern Gördes and the northern Çine submassifs are predicted to have been at temperatures in the lower apatite partialannealing zone or below. With the exception of the basement block in the Küçük Menderes graben, the Central Menderes metamorphic core complex remains at temperatures $>130^{\circ} \mathrm{C}$. The contoured temperature distribution in the northern Çine submassif is a little misleading because sample points are not evenly distributed but lie on two NNE-SSW transects in the western and eastern part of this submassif. Therefore, the relatively warm area in the central part of the northern Çine submassif is an artefact of the contouring procedure.

$14 \mathrm{Ma}$ : Cooling below temperatures of the apatite partial-annealing zone in the northern part of the Gördes submassif and the southern part of the Çine 

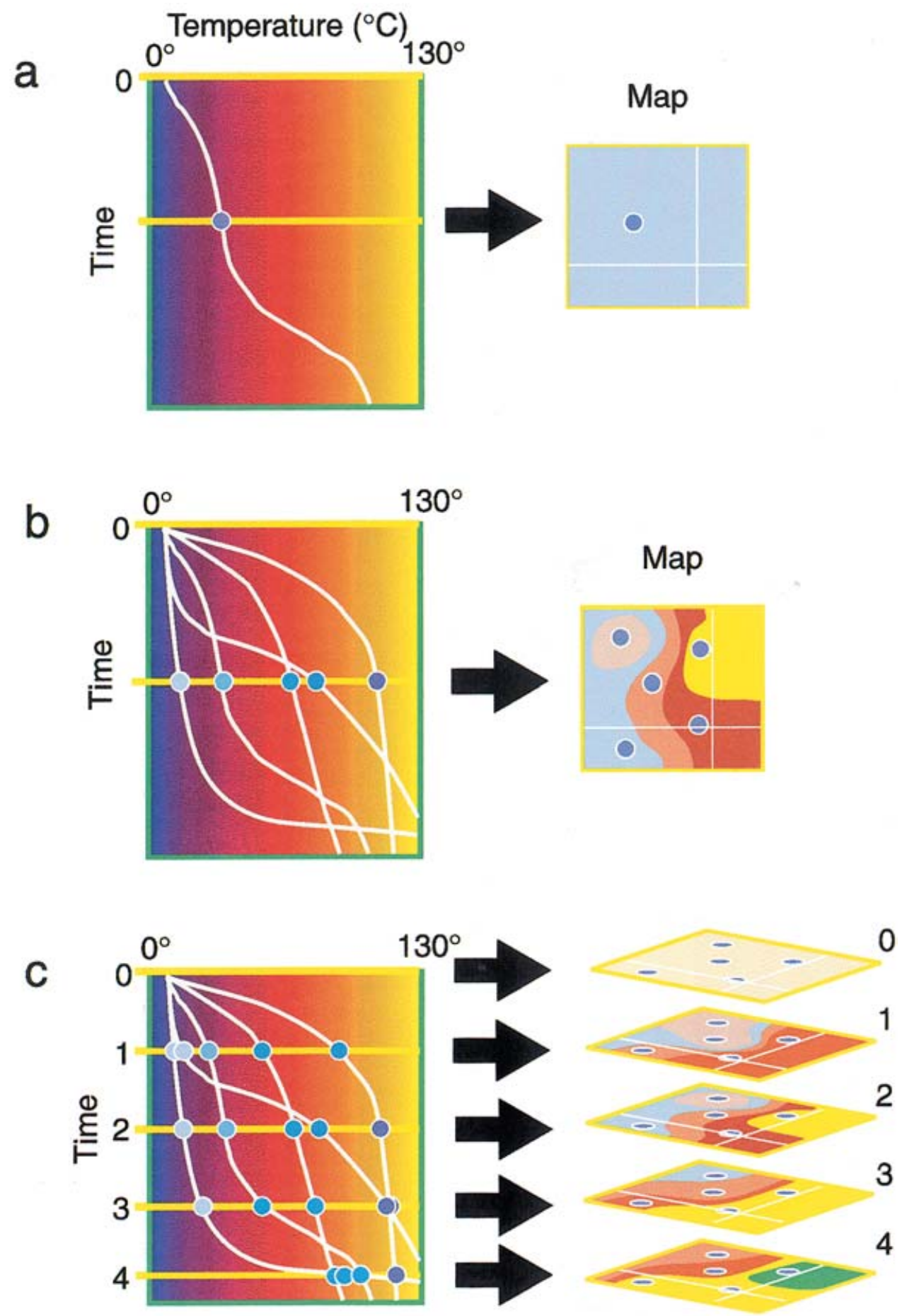

Figure 5. Converting thermal history information into images (from Gleadow et al. 1999). (a) Single sample yields thermal history such that for any given time a particular temperature (and its attendant uncertainty) can be attributed to geographic location. (b) Series of samples gives areal temperature distribution for a particular time, which can be contoured. (c) Series of time slices documenting regional temperature evolution.

submassif was completed. Temperatures throughout the Gördes and Çine submassifs were at surface values at this time, except a small warm spot in the southwestern Çine submassif, which is controlled by sample T84. During the 18-14 Ma period, cooling took place in the southeastern part of the Central Menderes metamorphic core complex. The contoured temperature distribution in the footwall of the Güney detachment is probably slightly misleading since the relatively cool samples in the Küçük Menderes graben in the centre of the Central Menderes metamorphic core complex and those from the southeastern part of the Central Menderes metamorphic core complex (samples T36, T40, T42, T44) control the map pattern. Sample 


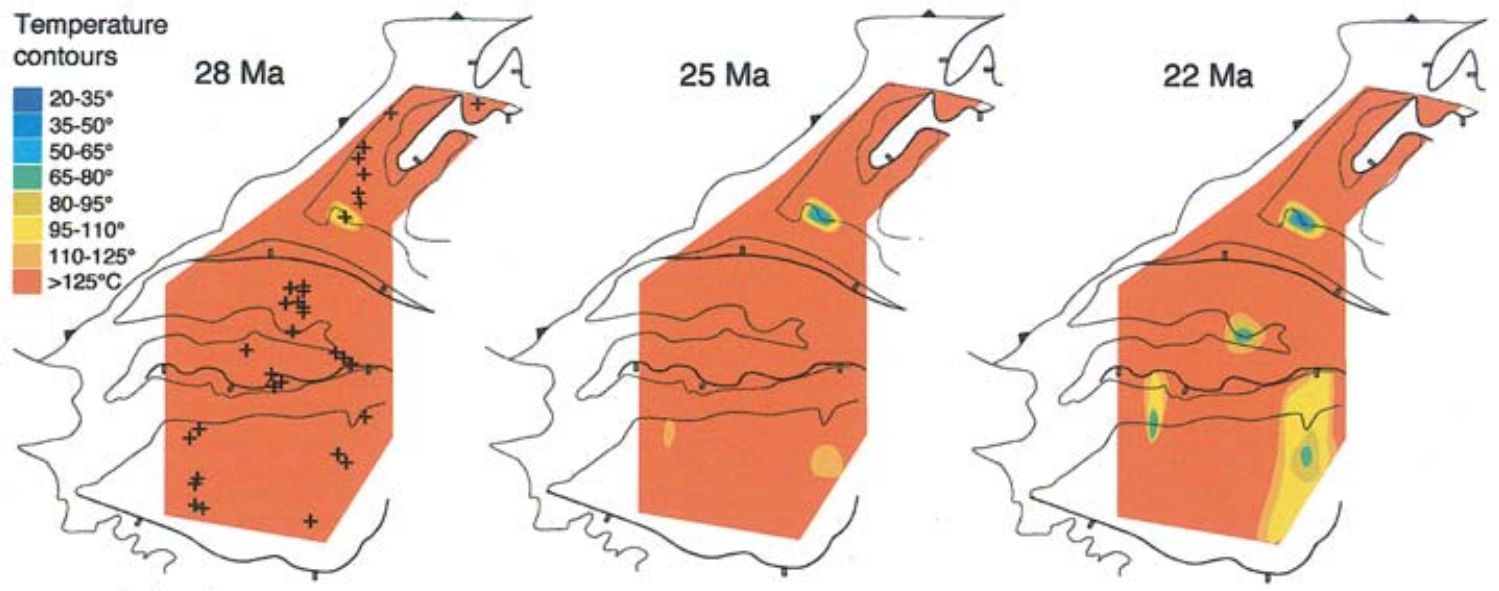

$+=$ sample locations
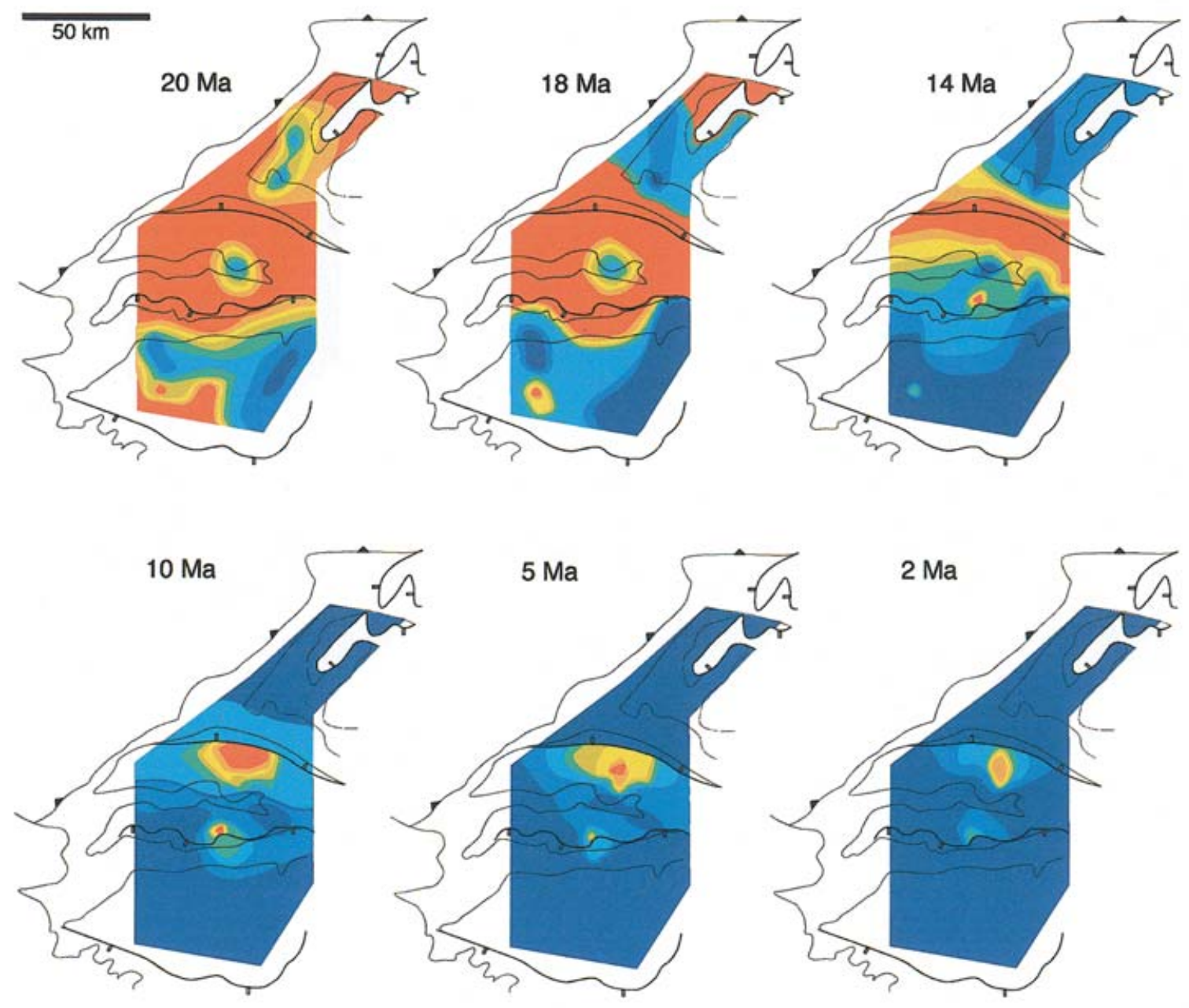

Figure 6. Time slices from $28 \mathrm{Ma}$ to $2 \mathrm{Ma}$ imposed on general outline of Menderes nappes with major detachments (Fig. 1); map for $28 \mathrm{Ma}$ shows locations of 34 samples used to construct maps; contours were drawn by computer program but were subsequently manipulated to include geological information, for example, hanging wall and footwall of detachments were not considered by computer-controlled contouring procedure, but manipulation ensured that cooling of footwall samples did not affect those of the hanging wall. Cooling below $\sim 130^{\circ} \mathrm{C}$ commenced in southern Gördes submassif at $\sim 28$ Ma but did not progress northward until $22-$ $20 \mathrm{Ma}$. Cooling in northern Çine submassif started at $25 \mathrm{Ma}$ and progressed southwards towards base of Lycian nappes. Down-faulted block in the central Central Menderes metamorphic core complex started to cool at $22 \mathrm{Ma}$. Footwall of Kuzey detachment remained at elevated temperatures above $\sim 130^{\circ} \mathrm{C}$ until $5 \mathrm{Ma}$; footwall of Güney detachment started to cool below $\sim 130^{\circ} \mathrm{C}$ at $14 \mathrm{Ma}$ but final cooling only occurred after 2 Ma. 
T35 from the immediate footwall of the central Güney detachment is still above $130^{\circ} \mathrm{C}$. This temperature is more likely to be typical of the footwall of the Güney detachment.

$10 \mathrm{Ma}$ : Cooling to temperatures below the apatite partial-annealing zone at the margins of the Küçük Menderes graben occurred. Temperatures in the southern segment of the Central Menderes metamorphic core complex remained at values of $\sim 80-90^{\circ} \mathrm{C}$. In the central part of the Central Menderes metamorphic core complex cooling into the apatite partial-annealing zone has occurred.

$5 \mathrm{Ma}$ : The northern flank of the Central Menderes metamorphic core complex below the Kuzey detachment remained at temperatures above $130^{\circ} \mathrm{C}$. The southern flank of the Central Menderes metamorphic core complex below the Güney detachment is also thought to be at temperatures $>80^{\circ} \mathrm{C}$, however, this inference is entirely based on sample T35.

$2 \mathrm{Ma}$ : The northern and southern flanks of the Central Menderes metamorphic core complex below the Güney and Kuzey detachments cool to similar temperatures of $\sim 60-80{ }^{\circ} \mathrm{C}$.

The final cooling to surface temperatures occurred during the interval 2-0 Ma in the footwalls of the Kuzey and Güney detachments.

\section{5.c. Summary of apatite fission-track data}

The onset of cooling from $>130$ to around $60^{\circ} \mathrm{C}$ recorded by the apatite fission-track data occurred during Late Oligocene times in the southern Gördes submassif, but this cooling is restricted to sample T68. Regional cooling in the Gördes submassif commenced at $\sim 20 \mathrm{Ma}$ and was completed by $\sim 14 \mathrm{Ma}$. In the Çine submassif regional cooling occurred during the same time span. The cooling pattern as derived from the apatite fission-track data supports top-to-the-S/SSW extension, not top-to-the-E extension and therefore supplies independent support of the conclusion by Régnier et al. (2003) that transport lineations were passively rotated within a relatively narrow zone at the base and directly underneath the Lycian nappes after tectonic movement (see Section 2.c). The central part of the Central Menderes metamorphic core complex also began cooling at this time. Nonetheless, with the exception of the down-faulted basement block in the Küçük Menderes graben, the present outcrop of the Central Menderes metamorphic core complex remained at temperatures greater than $130^{\circ} \mathrm{C}$ during this period. Cooling in the Gördes submassif appears to have migrated northwards towards the Simav detachment and the Izmir-Ankara zone, and southwards in the Çine submassif towards the base of the Lycian nappes.

From Late Miocene time onwards, a second phase of cooling from $>130$ to around $60^{\circ} \mathrm{C}$ affected much of the Central Menderes metamorphic core complex.
Cooling across the inner submassif was asymmetric with early cooling in the south. However, it appears that pronounced cooling in the immediate footwalls of the Kuzey and Güney detachments did not start until the Pliocene. Final cooling to surface temperatures of footwall rocks to the Güney and Kuzey detachments occurred during the Quaternary.

Although the cooling patterns appear to be locally asymmetric towards the major extensional detachments bounding the various submassifs, the overall finite pattern across the western Anatolide belt is remarkably symmetric.

\section{5.d. Regional cooling histories}

By combining the thermal histories inferred from apatite fission-track data with the zircon fission-track data (Table 1), the ${ }^{40} \mathrm{Ar}{ }^{39} \mathrm{Ar}$-muscovite ages shown in Fig. 4, and published ${ }^{40} \mathrm{Ar} /{ }^{39} \mathrm{Ar}$-muscovite and biotite ages (Hetzel et al. 1995a; Hetzel \& Reischmann, 1996; Lips et al. 2001), we illustrate the regional variations in the temperature history of the different submassifs (Fig. 7). The oldest cooling phase we can constrain with the data occurred in the southern Çine submassif (Fig. 7a). Initial cooling between $\sim 40 \mathrm{Ma}$ and $\sim 25 \mathrm{Ma}$ was slow $\left(\sim 10^{\circ} \mathrm{C} \mathrm{Myr}^{-1}\right)$. The distribution of the ${ }^{40} \mathrm{Ar} /{ }^{39} \mathrm{Ar}$-muscovite ages (Figs 3, 7a) suggest that cooling from maximum metamorphic temperatures of $\sim 500-550^{\circ} \mathrm{C}$ (Régnier et al. 2003) at the southern margin apparently progressed northward and structurally downward in the Çine submassif, indicating that the muscovite ages are cooling ages. More rapid cooling at rates greater than $30^{\circ} \mathrm{C} \mathrm{Myr}^{-1}$ occurred between $\sim 25$ and $18 \mathrm{Ma}$. In contrast to the northward younging ${ }^{40} \mathrm{Ar} /{ }^{39} \mathrm{Ar}$-muscovite ages, the apatite fission-track data show that shallow-level cooling progressed southward and structurally upward (Figs 2, 6).

The footwall of the Simav detachment cooled rapidly at rates greater than $50{ }^{\circ} \mathrm{C} \mathrm{Myr}^{-1}$ from after 25 to $20 \mathrm{Ma}$ (Fig. 7b). This inference is based on one single ${ }^{40} \mathrm{Ar} /{ }^{39} \mathrm{Ar}$-muscovite age and seven apatite fission-track ages.

The hanging wall of the Kuzey detachment (the southernmost Gördes submassif), the central Central Menderes metamorphic core complex and the hanging wall of the Güney detachment cooled rapidly at the Oligocene/Miocene boundary at rates greater than $50^{\circ} \mathrm{C} \mathrm{Myr}^{-1}$ (Fig. 7c-e). The presently exposed rocks of the Gördes and Çine submassifs and those in the central part of the Central Menderes metamorphic core complex were close to the surface in the Miocene (Fig. 7a-e).

In contrast, the footwall rocks of the Kuzey and Güney detachments remained at relatively high temperatures well after cooling to near surface temperatures had occurred in the two outer submassifs. After intrusion of the Salihli granite at $\sim 20 \mathrm{Ma}$, the rocks 


\section{Time (Ma)}

a Southern margin of Çine submassif

b Footwall of Simav detachment
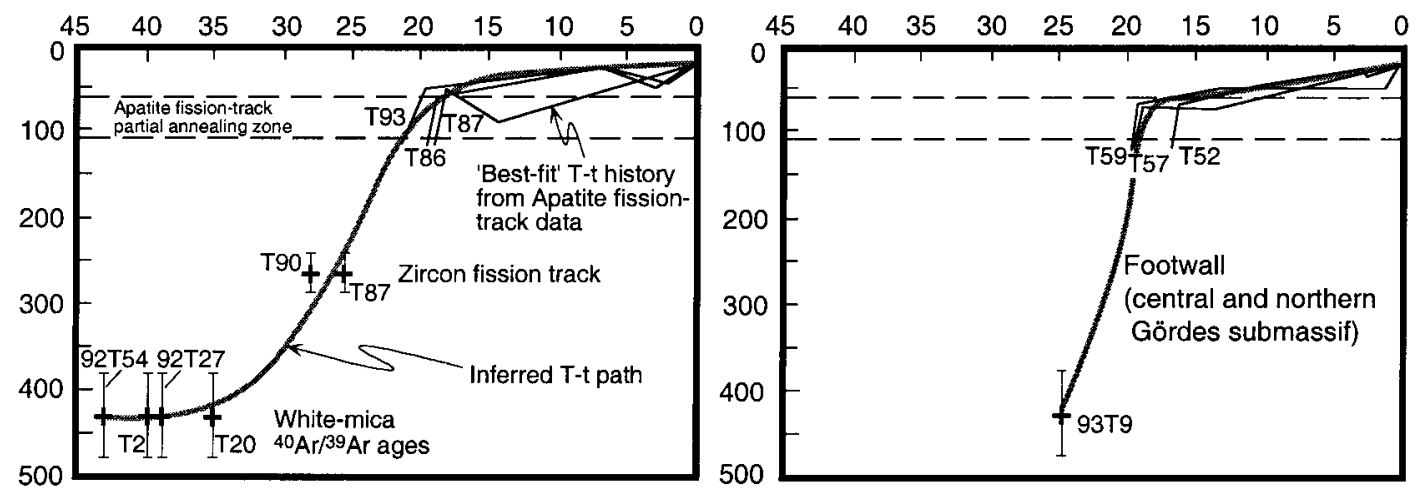

C Kuzey detachment

d Central CMCC
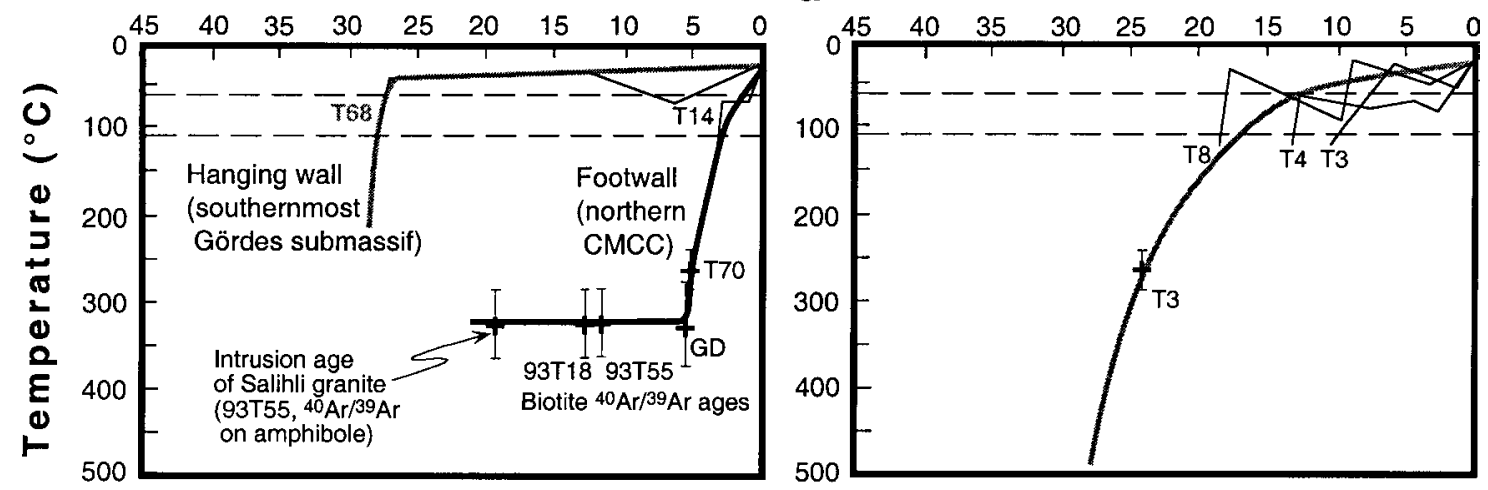

e Güney detachment

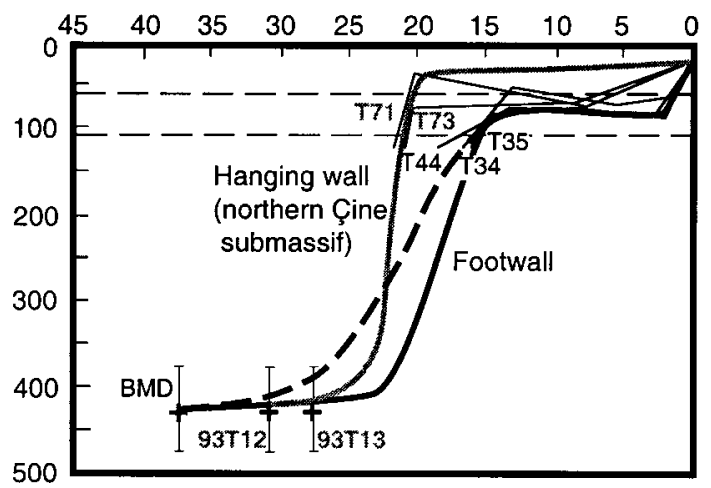

Figure 7. (a-e) Temperature-time diagrams for five areas along NNE-SSW cross-section (Figs 1, 2) combining ${ }^{40} \mathrm{Ar} /{ }^{39} \mathrm{Ar}-\mathrm{amphibole}$, muscovite and biotite, and apatite and zircon fission-track data; assumed closure temperature for $\mathrm{Ar}$ in muscovite is $\sim 400-450{ }^{\circ} \mathrm{C}$, $\sim 350^{\circ} \mathrm{C}$ for $\mathrm{Ar}$ in biotite, $240-280^{\circ} \mathrm{C}$ for fission tracks in zircon and $\sim 110 \pm 10^{\circ} \mathrm{C}$ for fluorapatite; intrusion age of Salihli granite and ${ }^{40} \mathrm{Ar} /{ }^{39} \mathrm{Ar}$-biotite data from Salihli granite are from Hetzel et al. $(1995 a) ;{ }^{40} \mathrm{Ar} /{ }^{39} \mathrm{Ar}$-muscovite data from samples 92T27, 92T54, T2 and T20 are from Hetzel \& Reischmann (1996) and those from samples GD and BMD are from Lips et al. (2001). Sample numbers in grey in (c) and (e) represent samples from hanging wall. $T-t$ histories for apatite were obtained from apatite fission-track data using Montetrax program of Gallagher (1995) which is based on apatite-annealing model of Laslett et al. (1987) and takes track-length distributions into account (note that for temperatures above $\sim 110^{\circ} \mathrm{C}$ and below $\sim 60^{\circ} \mathrm{C}$, apatite fission-track data provide no effective constraint on model evolution); whenever zircon fission-track or ${ }^{40} \mathrm{Ar} /{ }^{39} \mathrm{Ar}$-mica data were available, cooling curves were further refined by including those data. Çine (a) and Gördes submassifs (b and c) and also the central part of the Central Menderes metamorphic core complex (d) cooled in Early and Middle Miocene times to near surface temperatures, therefore Çine and Gördes submassifs can be viewed as being 'pinned' to Earth's surface, providing fixed framework in time and space to consider the subsequent emergence of the Central Menderes metamorphic core complex. Final cooling in footwalls of the Kuzey (c) and Güney detachments (e) occurred in Pliocene times; early cooling of footwall of the Güney detachment is poorly constrained, and dashed and non-dashed paths indicate alternative early cooling paths after metamorphic climax at 36-38 Ma (sample BMD). Note that rocks above and below the Güney detachment had a relatively similar cooling history in Late Oligocene and Early Miocene times, that is, before the Güney detachment formed, and that the poorly resolved Early/Middle Miocene cooling event in footwall of the Güney detachment coincides in time with $\mathrm{D}_{\mathrm{A} 4}$ extension during intrusion of Salihli and Turgutlu granites in the footwall of the Kuzey detachment. 
in the footwall of the Kuzey detachment did not cool to any significant degree until $\sim 5 \mathrm{Ma}$ when they started to cool rapidly at rates greater than $50^{\circ} \mathrm{C} \mathrm{Myr}^{-1}$ (Fig. 7c). Cooling in the footwall of the Güney detachment was more complex and involved a first poorly resolved phase in the Early/Middle Miocene followed by final cooling from shallow depth after $\sim 5 \mathrm{Ma}$ (Fig. 7e). The dashed and the non-dashed lines in Fig. 7e show two possible cooling paths for early cooling of the Güney footwall and highlight the problem in defining the early time-temperature history of the footwall of the Güney detachment.

\section{Discussion}

\section{6.a. Denudation of the outer submassifs}

In the Gördes submassif, the apatite fission-track ages decrease from $28 \mathrm{Ma}$ at the southern margin to $16 \mathrm{Ma}$ near the Simav graben in the north. However, sample T68 probably does not underlie the Simav detachment and therefore its cooling history, which is distinctly different from that of the other samples from the Gördes submassif, appears unrelated to movement on the Simav detachment. In the central part of the Gördes submassif, the apatite fission-track ages are virtually similar at $19-21 \mathrm{Ma}$ (Fig. 2). These cooling ages are the same as or slightly younger than those for the syn-detachment intrusion of the Eğrigöz granite at $20.7 \pm 0.5 \mathrm{Ma}$ (Ring \& Collins, 2003) and demonstrate fast Early Miocene cooling (Fig. 7b). Therefore, and because the apatite fissiontrack ages young northward in the direction of tectonic transport on the Simav detachment, cooling was related to movement on the Simav detachment.

A slip rate of $\sim 10 \mathrm{~km} \mathrm{Myr}^{-1}$ can be estimated from the inverse slope of apatite fission-track ages with distance in the slip direction (Fig. 2). Offset of geological markers suggest a minimum displacement of $\sim 50 \mathrm{~km}$ (Figs 1-3). Barometric estimates show that the total amount of exhumation since the Eocene peak of metamorphism in the Menderes nappes is of the order of 20-25 km (Ring et al. 1999a). Gessner et al. (2001a) showed that movement on the Kuzey and Güney detachments accounts for the final 10$12 \mathrm{~km}$ of this exhumation. Assuming that all of the earlier exhumation was due to detachment faulting, we can calculate a dip angle of the Simav detachment which, if displacement was assumed to be $50 \mathrm{~km}$, would be between $10^{\circ}$ and $20^{\circ}$. If a smaller displacement of $25 \mathrm{~km}$ was assumed, the dip angle would be of the order of $25-30^{\circ}$. If erosion and/or vertical ductile thinning acted as additional exhumation processes or displacement was greater than $50 \mathrm{~km}$, then the dip angle for the Simav detachment would be less. Although these estimates are not very accurate, the conclusion that the Simav detachment had an initially low dip angle, that is, less than $30^{\circ}$, appears to be robust.
In the Çine submassif, the apatite fission-track ages young southward from $\sim 22 \mathrm{Ma}$ in the north to $18 \mathrm{Ma}$ in the south (Fig. 2) and reveal a regional pattern strikingly similar to that in the footwall of the Simav detachment in the Gördes submassif. As the apatite fission-track cooling patterns are so similar, the cause of cooling may also be similar. This assumption has far-reaching implications because it demands the existence of an Early Miocene extensional detachment at the southern margin of the Çine submassif. However, as noted above, there is no well-defined extensional fault known from this area. Another problem is the geometry of the footwall of the demanded Lycian detachment. If the monocline in the footwall of the detachment represents a rolling hinge (Axen \& Bartley, 1997), a top-to-the$\mathrm{N}$ displacing detachment would be implied for which there is no evidence.

The cooling curve at the southern margin of the Çine submassif (Fig. 7a) indicates an initially slow cooling rate of $\sim 10^{\circ} \mathrm{C} \mathrm{Myr}^{-1}$ during Late Eocene and Oligocene times. The only major structure known from the Çine submassif that was active at this time is the Eocene Selimiye shear zone (Hetzel \& Reischmann, 1996). The northward- and structurally downwardyounging ${ }^{40} \mathrm{Ar} /{ }^{39} \mathrm{Ar}$-white-mica ages are compatible with top-to-the-S thrusting and are another strong indication that the Selimiye shear zone is a thrust and not an extensional fault as advocated by Bozkurt \& Park (1994). We relate slow cooling primarily to erosion after movement on the Selimiye shear zone. Erosion rates in this case would be of the order of $0.2-0.5 \mathrm{~km} \mathrm{Myr}^{-1}$. Acceleration of cooling to values greater than $30^{\circ} \mathrm{C} \mathrm{Myr}^{-1}$ coincides with the proposed Late Oligocene-Miocene extensional reactivation of the basal thrust of the Lycian nappes (Collins \& Robertson, 1998, 2003; Güngör \& Erdoğan, 2001). Our data indicate a Late Oligocene age for the onset of extension. The southward and structurally upward younging of the apatite fission-track data fits well with the interpretation that this phase of movement of the Lycian nappes was extensional and implies a slip rate of $\sim 4-5 \mathrm{~km} \mathrm{Myr}^{-1}$. Our general interpretation of cooling in the Çine submassif is that the fission-track ages are related to extensional faulting whereas the ${ }^{40} \mathrm{Ar} /{ }^{39} \mathrm{Ar}$ ages are not. This interpretation implies that the extensional fault roots at a relatively high crustal level, most probably at the brittle/ductile transition.

Cooling to near-surface temperatures in the southern part of the Gördes submassif and the northern part of the Çine submassif occurred in the Early to Middle Miocene and both outer submassifs subsequently formed plateau-like areas (Yilmaz et al. 2000). Presently flat-lying Early and Middle Miocene sedimentary rocks in the Gördes and Çine submassifs were deposited on basement, which has a regionally subhorizontal Eocene foliation. Afterwards, a Late Miocene, possibly Early Pliocene erosion surface formed on the flat-lying sediments on these plateaux 
(Yilmaz et al. 2000). The common subhorizontal orientation of the foliation, bedding in the basin sediments and the erosion surface indicates that the outer submassifs retained their Early Miocene geometry. Therefore, the subhorizontal structure of the Çine and Gördes submassifs can be viewed as being 'pinned' to the Earth's surface, providing a fixed framework in time and space to consider the subsequent emergence of the Central Menderes metamorphic core complex.

\section{6.b. Central Menderes metamorphic core complex}

The asymmetry of the cooling patterns across the Kuzey and Güney detachments and their associated graben indicates that the primary control on the unroofing of the Central Menderes metamorphic core complex was tectonic rather than erosional. Both the Kuzey and Güney detachments and the present-day Gediz and Büyük Menderes graben root below the corresponding Gördes and Çine submassifs (Cohen et al. 1995). This structural arrangement and the kinematic indicators during extensional deformation (Fig. 3), showing northerly hanging-wall transport in the northern Central Menderes metamorphic core complex (Hetzel et al. 1995a) and southerly directed hanging-wall transport in the southern Central Menderes metamorphic core complex (Emre \& Sözbilir, 1997), together with the temperature history information, support the contention that the Central Menderes metamorphic core complex underwent tectonic denudation that culminated in its exposure at the surface in the Pliocene and Quaternary.

Gessner et al. (2001a) showed that the gross synclinal structure and the brittle detachment systems in the central Central Menderes metamorphic core complex are related structures. Tectonic unloading along the detachments induced upward flexure of the upper crust. This rotated the presently exposed detachment surfaces into their present low angle of dip during progressive exhumation. The model of Gessner et al. (2001a) implies that the steep faults, which bound the Gediz and Büyük Menderes graben, formed as the two detachments were rotated into shallower orientations, that is, detachment and graben faults are related and manifest different stages of faulting and exhumation. Thermal modelling suggests that cooling related to formation of the Central Menderes metamorphic core complex started in the latest Miocene to Pliocene and seismic activity suggests that this process is still operating, that is, the Central Menderes metamorphic core complex is a nascent core complex. The estimated displacement of $\sim 10-12 \mathrm{~km}$ infers that the detachments operated at average slip rates of $\sim 2 \mathrm{~km} \mathrm{Myr}^{-1}$. The initial dip angle was of the order of $\sim 60^{\circ}$ for the Kuzey detachment and $\sim 40^{\circ}$ for the Güney detachment (Gessner et al. 2001a). Hence, the slip rates, the amount of displacement, the dip angles and the geometry of the footwalls for the two detachments that bound the Central Menderes metamorphic core complex are significantly different from those of the two outer detachments.

While the cooling history of the footwall of the Kuzey detachment is straightforward, the cooling history for the Güney detachment is more complex. This complexity might be an artefact caused by the poor data coverage, or by the fact that at the onset of cooling the southern margin of the Central Menderes metamorphic core complex had a lower geothermal gradient than that of the northern margin, which had been intruded by granitic magmas. In this case, the footwall of the Güney detachment may have had less potential to record a high-resolution cooling history. Another possibility might be that the poorly resolved early cooling history in the Miocene was related to movement on the proposed Lycian detachment. However, this scenario would demand a displacement of up to $100 \mathrm{~km}$ on the Lycian detachment. Hayward (1984) proposed that the Lycian nappes moved $\sim 70 \mathrm{~km}$ in Miocene times.

\section{6.c. Proposed denudation history and the symmetric cooling patterns}

A working hypothesis for the unroofing of the Anatolide belt is summarized in Fig. 8. Crustal shortening and suturing of the Izmir-Ankara zone with the rocks of the future Anatolide belt and southerly directed thrusting of the Lycian nappes and the Cycladic blueschist unit occurred from Late Cretaceous to Eocene times (Collins \& Robertson, 1998; Gessner et al. 2001c). A first phase of extension commenced in the Late Oligocene and Early Miocene and appears to be symmetric across the Anatolide belt. Relative displacement between the upper/middle and lower units and the Anatolide belt in the north was accommodated by the Simav detachment. We speculate that extension in the southern part of the belt was accommodated by top-to-the-S extensional movement at the base of the Lycian nappes. The early extensional phase resulted in fragmentation and thinning of the upper and middle units promoting cooling in the Gördes and Çine submassifs.

During Pliocene times, extension was transferred towards deeper structural levels of the Anatolide belt and the Kuzey and Güney detachments formed. Both detachments deformed the Late Miocene/Pliocene erosion surface, facilitated tectonic denudation of the Central Menderes metamorphic core complex until the present and also appear to have a symmetric regional arrangement.

Although the general Late Oligocene/Early Miocene cooling pattern is symmetric on a regional scale, we note that syn-extension granites are only exposed in the northern half of the Anatolide belt, that is, the Turgutlu and Salihli granites south of the Gediz graben and the Demirci, Koyunoba, Eğrigöz and minor 


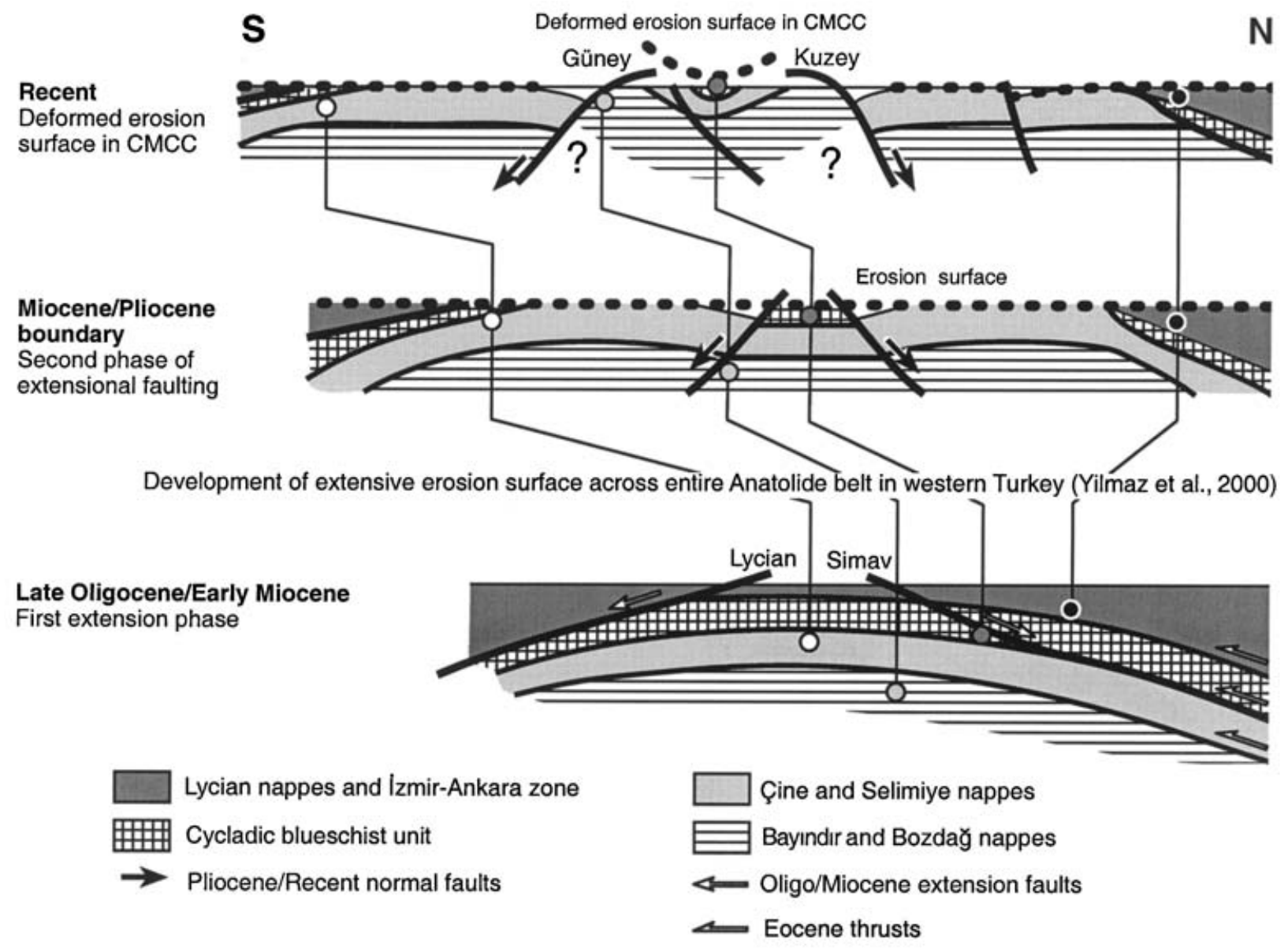

Figure 8. Simplified model for two-stage development of bivergent detachment systems. First phase of extensional faulting in Late Oligocene and Early Miocene times reactivated pre-existing faults and therefore occurred on low-angle structures and was followed by formation of extensive Late Miocene/Pliocene erosion surface (Yilmaz et al. 2000). Erosion surface was subsequently deformed by Kuzey and Güney detachments, which formed at the Miocene/Pliocene boundary; Kuzey and Güney detachments are rolling-hinge fault systems, which formed at high angles to Earth's surface (Gessner et al. 2001a).

related granites in the northern Gördes submassif. An additional problem with the Turgutlu and Salihli granites is that they were syn-tectonic with respect to the ductile $\mathrm{D}_{\mathrm{A} 4}$ extensional event at $\sim 18-20 \mathrm{Ma}$ (Hetzel et al. 1995a), but this extensional event did not cause any significant cooling of the granites (Fig. 7c). The timing of the $\mathrm{D}_{\mathrm{A} 4}$ extensional event in the northern Central Menderes metamorphic core complex fits well with the timing for the Simav detachment and it might be feasible to assume that a small-scale extensional shear zone formed in the footwall of the Simav detachment. However, this interpretation would demand a displacement of more than $100 \mathrm{~km}$ on the Simav detachment. If it was accepted that cooling of sample T68 from the southernmost Gördes submassif was not related to movement on the Simav detachment, then the Simav detachment was not directly associated with intrusion of the Turgutlu and Salihli granites. Another deviation from the overall symmetry of extensional faulting is that the Simav detachment in the north is associated with extensive basin development, whereas in the south, Miocene basins are more limited.
Was the denudation history of the western Anatolide belt the product of two distinct events, or did it arise from one single extension event that progressed more or less continuously from higher towards deeper structural levels? We argue that the cooling curves suggest two distinct events that were separated in time by $\sim 15 \mathrm{Myr}$. There is growing evidence that detachments are shortlived (up to $\sim 5 \mathrm{Myr}$ ) and relatively rapidly operating structures triggered by tectonic pulses (e.g. Lister \& Forster, 1996; Ring, Thomson \& Bröcker, 2003). The different tectonic characteristics of the two outer detachment systems as compared to the detachments bounding the Central Menderes metamorphic core complex are in line with our inference that they were caused by two separate events.

The reactivation of the Cyclades-Menderes thrust and the basal thrust of the Lycian nappes during the first extension event appears to be very important for the formation of the detachment as low-angle faults. Low-angle detachments in the Aegean also reactivated former thrust planes (e.g. the Cretan, the Ios and the Vari detachment). The Kuzey and Güney detachments 
formed at high angles and cut across pre-existing structures.

Finally, it should be noted that the regional cooling and extension pattern in the Menderes nappes of the Anatolide belt in western Turkey must not be the only extensional structures. We showed that extensional faulting and associated cooling progressed structurally downward towards the centre of the Anatolide belt, suggesting that older normal faults should be searched for in the upper tectonic units. Gessner et al. (2001c) discussed the possibility of an Eocene top-to-theNE ductile normal shear zone within the Cycladic blueschist unit.

\section{6.d. Comparisons with the pattern of extensional faults in the central and southern Aegean Sea}

The pattern of top-to-the-N and top-to-the-S extensional faults in the Anatolide belt of western Turkey is more or less symmetric at the orogen scale. Accordingly, the pattern of apatite fission-track ages also has a symmetric arrangement at this scale. In contrast, the nearby Aegean is characterized by more uniform topto-the-N extensional faults, with top-to-the-E extension on Samos, the top-to-the-S Ios detachment and the little-known top-to-the-S detachment on Serifos Island being the only known exceptions (Lee \& Lister, 1992; Buick, 1991; Lister \& Forster, 1996; Forster \& Lister, 1999; Thomson, Stöckhert \& Brix, 1999; Ring, Laws \& Bernet, 1999; Grasemann et al. 2002; Ring \& Reischmann, 2002). The apatite fission-track ages reported by Altherr et al. (1982), Thomson, Stöckhert \& Brix (1999) and Ring, Thomson \& Bröcker (2003) from the Aegean appear to young northward from $\sim 13-20 \mathrm{Ma}$ on the island of Crete to $\sim 8-12 \mathrm{Ma}$ on the islands in the Cyclades. This northward-younging trend agrees with the admittedly poorly constrained ages for the extensional detachments that primarily caused the cooling. The Cretan detachment, which probably underlies much of the southern Aegean (Ring $\&$ Reischmann, 2002) operated at $\sim 20 \mathrm{Ma}$ (Thomson, Stöckhert \& Brix, 1999), the Ios detachment at $\sim 12 \mathrm{Ma}$ (Henjes-Kunst \& Kreuzer, 1982) and the Montsuna detachment on Naxos and the Vari detachment on Syros and Tinos at 9-12 Ma (Lister \& Forster, 1996; Ring, Thomson \& Bröcker, 2003) (Fig. 1, inset). The differences in the pattern of detachment faults and associated cooling between the Aegean and the western Anatolide belt underscores that there are major alongstrike differences in the Hellenide-Anatolide orogen and that the cause for extension in both areas was different.

In the Aegean the southward retreating Hellenic subducting slab is widely envisioned to be the cause of detachment faulting (Lister, Banga \& Feenstra, 1984; Buick, 1991; Thomson, Stöckhert \& Brix, 1999; Ring \& Layer, 2003). Hetzel et al. (1995b) proposed that the collision of the exotic Anatolide microcontinent in Eocene times halted subduction in the Anatolide belt. Hetzel et al. (1995b) proposed that differences in potential energy associated with thermal relaxation caused Late Oligocene/Early Miocene extension in the Anatolide belt. The younger phase of extensional faulting in the 'inner' submassif and the formation of the Central Menderes metamorphic core complex shows pronounced differences to the older extensional event. Şengör (1987) suggested that the westward escape of Anatolia and the formation of the North and South Anatolian strike-slip faults triggered late N-S extension in western Turkey from latest Miocene times onwards. Burchfiel et al. (2000) argued that the North Anatolian fault had its inception in western Turkey and the northern Aegean Sea at $\sim 4-5 \mathrm{Ma}$ and this timing would fit with the inception of detachment faulting and the formation of the Central Menderes metamorphic core complex.

\section{Conclusions}

Detachment-related denudation and cooling to near surface temperatures occurred over a $\sim 25 \mathrm{Myr}$ time interval in the Anatolide belt of western Turkey and is presently still active. The inferred cooling histories show a remarkably symmetric pattern across the Anatolide belt and progressed in two stages from the outer submassifs towards the centre of the belt, where extension is today focused. Combining the temperature-time history of the Central Menderes metamorphic core complex with structural data points to the conclusion that detachment faulting from Pliocene times to the present controlled the cooling history of this central section of the Anatolide belt. Extensional faulting also controlled earlier cooling in the Gördes and Çine submassifs. A hypothesis has been put forward, which explains cooling in these two submassifs as a consequence of Late Oligocene to Early Miocene normal faulting and thinning of a crustal-scale hanging wall consisting of the Cycladic blueschist unit, the Lycian nappes and the Izmir-Ankara zone.

Acknowledgements. This study was funded by the Deutsche Forschungsgemeinschaft (grants Ri538/4 and Ri 538/15). We thank Talip Güngör for his extensive help and support during field work in Turkey and Alan Collins, Kerry Gallagher and Roland Oberhänsli for reviews.

\section{References}

Altherr, R., KreuZer, H., Wendt, I., Lenz, H., WAGNER, G. H., Keller, J., HARRE, W. \& HöHNDORF, A. 1982. A late Oligocene/early Miocene high temperature belt in the Attic-Cycladic crystalline complex (SE Pelagonian, Greece). Geologisches Jahrbuch E23, 97-164.

AXEN, G. J. \& BARTLEY, J. M. 1997. Field tests of rolling hinges: Existence, mechanical types, and implications for extensional tectonics. Journal of Geophysical Research 102, 20515-37.

BOzKURT, E. 2000. Timing of extension on the Büyük Menderes Graben, western Turkey, and its tectonic 
implications. In Tectonics and Magmatism in Turkey and the Surrounding Area (eds E. Bozkurt, J. A. Winchester and J. D. A. Piper), pp. 385-403. Geological Society of London, Special Publication no. 173.

BOzKURT, E. \& PARK, R. G. 1994. Southern Menderes Massif: an incipient metamorphic core complex in western Anatolia, Turkey. Journal of the Geological Society, London 151, 213-16.

Bradbury, H. J. \& Nolen-HoEksema, R. C. 1985. The Lepontine Alps as an evolving metamorphic core complex during A-type subduction: Evidence from heat flow, mineral cooling ages, and tectonic modeling. Tectonics 4, 187-211.

Brandon, M. T., Roden-Tice, M. K. \& GARVER, J. I. 1998. Late Cenozoic exhumation of the Cascadia accretionary wedge in the Olympic Mountains, NW Washington State. Geological Society of America Bulletin 110, 9851009.

BuCHER, K. \& FreY, M. 1994. Petrogenesis of metamorphic rocks, 6th ed. Berlin: Springer, $318 \mathrm{pp}$.

BUICK, I. S. 1991. The late-Alpine evolution of an extensional shear zone, Naxos, Greece. Journal of the Geological Society, London 152, 639-54.

BurChFIEL, C. B., NAKOV, R., TZANKOV, T. \& ROYDEN, L. H. 2000. Cenozoic extension in Bulgaria and northern Greece: the northern part of the Aegean extensional regime. In Tectonics and Magmatism in Turkey and the Surrounding Area (eds E. Bozkurt, J. A. Winchester and J. D. A. Piper), pp. 325-52. Geological Society of London, Special Publication no. 173.

ÇAĞLAYAN, M. A., ÖZTÜRK, E. M., ÖZTÜRK, S., SAV, H. \& AKAT, U. 1980. Menderes Masifi güneyine ait bulgular ve yapisal yorum. Geological Engineering 10, 9-19.

CANDAN, O., DORA, Ö. O., OBERHÄNSLI, R., ÇETINKAPLAN, M., Partzisch, J. H., Warkus, F. C. \& DÜrR, S. 2001. Pan-African high-pressure metamorphism in the Precambrian basement of the Menderes Massif, western Anatolia, Turkey. International Journal of Earth Sciences 89, 793-807.

CANDAN, O., DORA, Ö. O., OBERHÄNSLI, R., OELSNER, F. \& DÜRR, S. 1997. Blueschist relics in the Mesozoic cover series of the Menderes Massif and correlations with Samos Island, Cyclades. Schweizerische Mineralogische und Petrographische Mitteilungen 77, 95-9.

COHEN, H. A., DART, C. J., AKYÜZ, H. S. \& BARKA, A. 1995. Syn-rift sedimentation and structural development of Gediz and Büyük Menderes Grabens, western Turkey. Journal of the Geological Society, London 152, 629-38.

Collins, A. S. \& Robertson, A. H. F. 1997. Lycian melange, southerwestern Turkey: An emplaced Late Cretaceous accretionary complex. Geology 25, 255-8.

Collins, A. S. \& Robertson, A. H. F. 1998. Processes of Late Cretaceous to Late Miocene episodic thrust sheet translation in the Lycian Taurides, SW Turkey. Journal of the Geological Society, London 155, 759-72.

Collins, A. S. \& Robertson, A. H. F. 2003. Kinematic evidence fore late Mesozoic-Miocene emplacement of the Lycian Allochthon over the western Anatolide belt, SW Turkey. Geological Journal (in press).

DANNAT, C. \& REISCHMANN, T. 1999. Single zircon ages of migmatites from the Menderes Massif, SW Turkey. Journal of Conference Abstracts 4, 805.

EMre, T. \& SÖZBILIR, H. 1997. Field evidence for metamorphic core complex, detachment faulting and accommodation faults in the Gediz and Büyük Menderes grabens, western Anatolia. International Earth Science
Colloquium on the Aegean and surrounding regions 1995, Proceedings 1, 73-94.

ENGEL, M. \& REISCHMANN, T. 1998. Single zircon geochronology of orthogneisses from Paros, Greece. Bulletin of the Geological Society of Greece 32, 91-9.

Fitzgerald, P. G., Sorkhabi, R. B., Redfield, T. F. \& STUMP, E. 1995. Uplift and denudation of the central Alaska Range: A case study in the use of apatite fission track thermochronology to determine absolute uplift parameters. Journal of Geophysical Research 100, 20175-91.

Foster, D. A., Gleadow, A. J. W., Reynolds, S. J. \& FITZGERALD, P. G. 1993. Denudation of metamorphic core complexes and the reconstruction of the transition zone, west central Arizona: Constraints from apatite fission track thermochronology. Journal of Geophysical Research 98, 2167-85.

Foster, D. A. \& John, B. E. 1999. Quantifying tectonic exhumation in an extensional orogen with thermochronology: examples from the southern Basin and Range province. In Exhumation processes: Normal faulting, ductile flow and erosion (eds U. Ring, M. T. Brandon, G. S. Lister and S. D. Willett), pp. 34364. Geological Society of London, Special Publication no. 154.

Foster, D. A., Miller, D. S. \& Miller, C. F. 1991. Tertiary extension in the Old Women Mountains area, California: Evidence from apatite fission track analysis. Tectonics 10, 875-86.

Forster, M. \& Lister, G. S. 1999. Detachment faults in the Aegean core complex of Ios, Cyclades, Greece. In Exhumation processes: Normal faulting, ductile flow and erosion (eds U. Ring, M. T. Brandon, G. S. Lister and S. D. Willett), pp. 305-24. Geological Society of London, Special Publication no. 154.

Fytikas, M., InNOCEnTI, F., MANetTI, P., Mazzuoli, R., Peccerillo, A. \& Villari, L. 1984. Tertiary to Quaternary evolution of volcanism in the Aegean region. In The geological evolution of the eastern Mediterranean (eds A. H. F. Robertson and J. E. Dixon), pp. 687-99. Geological Society of London, Special Publication no. 17.

Galbraith, R. F. \& Laslett, G. M. 1993. Statistical models for mixed fission track ages. Nuclear Tracks and Radiation Measurements 21, 459-70.

GALlAGHER, K. 1995. Evolving temperature histories from apatite fission track data. Earth and Planetary Science Letters 136, 421-35.

Gessner, K., Collins, A. S., Ring, U. \& GÜNGÖR, T. 2003. Resolving the structural and thermal history of poly-orogenic basement: SHRIMP geochronology of granitoid rocks in the southern Anatolide belt, western Turkey. Journal of the Geological Society, London, in press.

GeSSNER, K., PIAZOLO, S., GÜNGÖR, T., RING, U., KRÖNER, A. \& PASSCHIER, C. W. 2001b. Tectonic significance of deformation patterns in granitoid rocks of the Menderes nappes, Anatolide belt, southwest Turkey. International Journal of Earth Sciences 89, 766-80.

Gessner, K., Ring, U., Johnson, C., Hetzel, R., PASSCHIER, C. W. \& GÜNGÖR, T. 2001a. An active bivergent rolling-hinge detachment system: The central Menderes metamorphic core complex in western Turkey. Geology 29, 611-14.

Gessner, K., RING, U., PAsschier, C. W. \& GÜNGÖR, T. 2001c. How to resist subduction: evidence for 
large-scale out-of-sequence thrusting during Eocene collision in western Turkey. Journal of the Geological Society, London 158, 769-84.

GLEADOW, A. J. W. \& DUDDY, I. R. 1981. A natural long-term track annealing experiment on apatite. Nuclear Tracks $5,169-74$.

Gleadow, A. J. W., Kohn, B. P., Brown, R. W. \& Gallagher, K. 1999. Regional imaging of denudation histories: new directions using low-temperature thermochronology. EOS, Transactions of the American Geophysical Union 80(46), 1038.

Grasemann, B., Zamolyi, A., Petrakakis, K., RAMBOUSEK, C. \& IGElSEDER, C. 2002. A new metamorphic core complex in the West cyclades (Serifos, Greece). Erlanger Geologische Abhandlungen 3, 36-7.

GÜNGÖR, T. \& ERDOǦAN, B. 2001. Emplacement age and direction of the Lycian nappes in the Söke-Selçuk region, western Turkey. International Journal of Earth Sciences 89, 870-82.

HAYWARD, A. B. 1984. Miocene clastic sedimentation related to the emplacement of the Lycian Nappes and the Antalya Complex. In The geological evolution of the eastern Mediterranean (eds A. H. F. Robertson and J. E. Dixon), pp. 287-300. Geological Society of London, Special Publication no. 17.

Henjes-Kunst, F. \& KReuZER, H. 1982. Isotopic dating of pre-alpidic rocks from the island of Ios (Cyclades, Greece). Contributions to Mineralogy and Petrology $\mathbf{8 0}$, 245-53.

Hetzel, R., PASSchier, C. W., Ring, U. \& DorA, Ö. O. 1995b. Bivergent extension in orogens: The Menderes Massif (southwestern Turkey). Geology 23, 455-8.

Hetzel, R. \& Reischmann, T. 1996. Intrusion age of Pan-African augen gneiss in the southern Menderes Massif and the age of cooling apatite fission-tracker Late Cretaceous-Tertiary ductile extensional deformation. Geological Magazine 133, 565-72.

Hetzel, R., Ring, U. Akal, C. \& Troesch, M. $1995 a$. Miocene NNE-directed extensional unroofing in the Menderes Massif, southwestern Turkey. Journal of the Geological Society, London 152, 639-54.

Hetzel, R., Romer, R. L., Candan, O. \& Passchier, C. W. 1998. Geology of the Bozdag area, central Menderes Massif, SW Turkey: Pan-African basement and Late Cretaceous-Tertiary deformation. Geologische Rundschau 87, 394-406.

HURFORD, A. J. 1986. Cooling and uplift patterns in the Lepontine Alps, south central Switzerland, and an age of vertical movement on the Insubric fault line. Contributions to Mineralogy and Petrology 93, 413-27.

HURFORD, A. J. 1990. Standardization of fission-track dating calibration: Recommendation by the Fission Track Working Group of the I. U. G. S. Subcommission on Geochronology. Chemical Geology 80, 171-8.

HURFORD, A. J., FLISCH, M. \& JÄGER, E. 1989. Unravelling the thermo-tectonic evolution of the Alps: A contribution from fission track analysis and mica dating. In Late Cretaceous-Tertiary Tectonics (eds M. P. Cowan, D. Dietrich and R. G. Park), pp. 369-98. Geological Society of London, Special Publication no. 45.

HURFORD, A. J. \& GREEN, P. F. 1982. A users' guide to fission track dating. Earth and Planetary Science Letters 59, 343-54.
IŞIK, V. \& TEKELI, O. 2001. Late orogenic crustal extension in northern Menderes Massif (western Turkey): evidence for metamorphic core complex formation. International Journal of Earth Sciences 89, 757-65.

JOHNSON, C. 1997. Resolving denudational histories in orogenic belts with apatite fission track thermochronology and structural data: An example from southern Spain. Geology 25, 623-6.

Johnson, C., Harbury, N. \& Hurford, A. J. 1997. The role of extension in the Miocene denudation of the Nevado-Filábride Complex, Betic Cordillera, SE Spain. Tectonics 16, 189-204.

Laslett, G. M., GReEN, P. F., DudDy, I. R. \& Gleadow, A. J. W. 1987. Thermal annealing of fission tracks in apatite. 2. A quantitative discription. Chemical Geology $\mathbf{6 5}, 1-13$.

LEE, J. \& LiSTER, G. S. 1992. Late Miocene ductile extension and detachment faulting, Mykonos, Greece. Geology 20, $121-4$.

Lips, A. L. W., Cassard, D., Sözbilir, H., Yilmaz, H. \& WiJBRANS, J. R. 2001. Multistage exhumation of the Menderes Massif, western Anatolia (Turkey). International Journal of Earth Sciences 89, 781-92.

Lister, G. S., Banga, G. \& Feenstra, A. 1984. Metamorphic core complexes of Cordilleran type in the Cyclades, Aegean Sea, Greece. Geology 12, 221-5.

Lister, G. S. \& Forster, M. 1996. Inside the Aegean metamorphic core complexes. Technical Publications of the Australian crustal research centre 45, $110 \mathrm{pp}$.

Loos, S. \& ReISCHMANN, T. 1999. The evolution of the southern Menderes Massif in SW Turkey as revealed by zircon dating. Journal of the Geological Society, London 156, 1021-30.

Oberhänsli, R., PARTzsch, J. H., CANDAN, O. \& ÇETIKAPlAN, M. 2001. First occurrence of Fe-Mg carpholite documenting a high-pressure metamorphism in metasediments of the Lycian Nappes, SW Turkey. International Journal of Earth Sciences 89, 867-73.

RÉGNIER, J.-L., RING, U., PASSCHIER, C. W. \& GÜNGÖR, T. 2003. Contrasting metamorphic evolution of metasediments from the Çine and Selimiye nappes in the southern Anatolide belt, western Turkey. Journal of Metamorphic Geology, in press.

ReIsChMANN, T. 1998. Pre-Alpine origin of tectonic units from the metamorphic complex of Naxos, Greece, identified by single zircon $\mathrm{Pb} / \mathrm{Pb}$ dating. Bulletin of the Geological Society of Greece 32, 101-11.

Reischmann, T., Kostopoulos, D. K., Loos, S., Anders, B., Avgerinas, A. \& Sklavounos, S. A. 2001. Late Paleozoic magmatism in the basement rocks southwest of Mt. Olympos, central Pelagonian zone, Greece: Remnants of a Permo-Carboniferous magmatic arc. Bulletin of the Geological Society of Greece 34, 985-94.

Ring, U., Brachert, T. C. \& Fassoulas, C. 2001. Middle Miocene graben development in Crete and its possible relation to large-scale detachment faults. Terra Nova 13, 297-304.

Ring, U., Brandon, M. T., Willett, S. D. \& Lister, G. S. 1999b. Exhumation processes. In Exhumation processes: Normal faulting, ductile flow and erosion (eds U. Ring, M. T. Brandon, G. S. Lister and S. D. Willett), pp. 1-27. Geological Society of London, Special Publication no. 154. 
Ring, U. \& Collins, A. S. 2003. SHRIMP dating of the syn-tectonic Egrigöz granite: Precise timing of corecomplex formation in the Anatolide belt of western Turkey. Terra Nova, in press.

Ring, U., Gessner, K., GÜNGÖr, T. \& PAsschier, C. W. 1999a. The Menderes belt of western Turkey and the Cycladic belt in the Aegean - do they really correlate? Journal of the Geological Society, London 156, 3-6.

RING, U., LAWS, S. \& BERNET, M. 1999. Structural analysis of a complex nappe sequence and late-orogenic basins from the Aegean Island of Samos, Greece. Journal of Structural Geology 21, 1575-1601.

RING, U. \& LAYER, P. W. 2003. High-pressure metamorphism in the Aegean, eastern Mediterranean: sequential accretion from the Late Cretaceous until the Miocene to Recent. Tectonics 22(3), 1022, DOI: 10.1029/2001TC001350, 23 p.

Ring, U., LAYER, P. W. \& ReISCHMANN, T. 2001. Miocene high-pressure metamorphism in the Cyclades and Crete, Aegean Sea, Greece: Evidence for large-magnitude displacement on the Cretan detachment. Geology 29, 395-8.

RING, U. \& REISCHMANN, T. 2002. The weak and superfast Cretan detachment, Greece: Exhumation at subduction rates in extrusion wedges. Journal of the Geological Society, London 159, 225-8.

Ring, U., ThOMsOn, S. N. \& BRÖCKER, M. 2003. Fast extension but little exhumation: The Vari detachment in the Cyclades, Greece. Geological Magazine 130, 245-52.

Ring, U., Willner, A. \& LACKMANN, W. 2001. Nappe stacking and clockwise versus anticlockwise pressuretemperature paths: an example from the Menderes nappes of western Turkey. American Journal of Science 301, 912-44.

ROBERTSON, A. H. F. \& DiXON, J. E. 1984. Introduction: aspects of the geological evolution of the Eastern Mediterranean. In The geological evolution of the Eastern Mediterranean (eds J. E. Dixon and A. H. F. Robertson), pp. 1-74. Geological Society of London, Special Publication no. 17.

Sherlock, S., Kelley, S., Inger, S., Harris, N. \& OKAY, A. $1999 .{ }^{40} \mathrm{Ar}-{ }^{39} \mathrm{Ar}$ and $\mathrm{Rb}-\mathrm{Sr}$ geochronology of highpressure metamorphism and exhumation history of the Tavşanli Zone, NW Turkey. Contributions to Mineralogy and Petrography 137, 46-58.

SCHUILING, R. D. 1962. On petrology, age, and structure of the Menderes migmatite complex (SW-Turkey). Bulletin of the Mineral Resources and Exploration Institute of Turkey 58, 71-84.

ŞENGÖR, A. M. C. 1987. Cross faults and differential stretching in their hanging walls in regions of low-angle normal faulting: examples from western Turkey. In Continental extensional tectonics (eds M. J. Coward, J. F. Dewey and P. L. Hancock), pp. 40573. Geological Society of London, Special Publication no. 28 .

SeyitoĞlu, G., Scott, B. C. \& Rundle, C. C. 1992. Timing of Cenozoic extensional tectonics in west Turkey. Journal of the Geological Society, London 149, 533-8.

SEYITOĞLU, G. \& SCOTT, B. C. 1996. The age of the Alaşehir graben (west Turkey) and its tectonic implications. Geological Journal 31, 1-11.

Tagami, T. \& Dumitru, T. A. 1996. Provenance and thermal history of the Francsican accretionary complex: Constraints from zircon fission track thermochronology. Journal of Geophysical Research 101, 11353-64.

Thomson, S. N., STÖCKHERT, B. \& BRIX, M. A. 1999. Miocene high-pressure metamorphic rocks of Crete, Greece: Rapid exhumation by buoyant escape. In Exhumation processes: Normal faulting, ductile flow and erosion (eds U. Ring, M. T. Brandon, G. S. Lister and S. D. Willett), pp. 87-108. Geological Society of London, Special Publication no. 154.

VILLA, I. M. \& PUXEDDU, M. 1994. Geochronology of the Larderello geothermal field: new data and the "closure temperature" issue. Contributions to Mineralogy and Petrology 115, 415-26.

WAgner, G. A. \& ReIMER, G. M. 1972. Fission track tectonics: the tectonic interpretation of fission track ages. Earth and Planetary Science Letters 14, 263-8.

WHEeler, J. \& ButleR, R. W. H. 1994. Criteria for identifying structures related to true crustal extension in orogens. Journal of Structural Geology 16, 1023-8.

WhitNEY, D. L. \& BOZKURT, E. 2002. Metamorphic history of the southern Menderes Massif, Western Turkey. Geological Society of America Bulletin 114, 829-38.

YILMAZ, Y., GENÇ, Ş. C., GÜRER, Ö. F., BOZCU, M., YILMAZ, K., Karacik, Z., Altunkaynak, S. \& Elmas, A. 2000. When did the western Anatolian grabens begin to develop? In Tectonics and Magmatism in Turkey and the Surrounding Area (eds E. Bozkurt, J. A. Winchester and J. D. A. Piper), pp. 353-84. Geological Society of London, Special Publication no. 173. 